\title{
Mobility management for multi-user sessions in next generation wireless systems
}

\author{
Eduardo Cerqueira ${ }^{\mathrm{a}, *}$, Luis Veloso ${ }^{\mathrm{a}}$, Augusto Neto ${ }^{\mathrm{a}}$, Marilia Curado ${ }^{\mathrm{a}}$, \\ Edmundo Monteiro ${ }^{\mathrm{a}}$, Paulo Mendes ${ }^{\mathrm{b}, 1,2}$ \\ a University of Coimbra, Informatics Engineering, Polo II, Pinhal de Marrocos, 3030-290 Coimbra, Portugal \\ ${ }^{\mathrm{b}}$ INESC Porto, Rua Dr. Roberto Frias, 378, 4200-465 Porto, Portugal
}

Available online 28 December 2007

\begin{abstract}
Mobility management and ubiquitous access for real-time multi-user sessions with Quality of Service (QoS) support are major requirements to the success of next generation wireless systems. In this context, Multi-User Session Control (MUSC) is proposed to allow fixed and mobile users to access multi-user sessions ubiquitously, while providing QoS mapping, QoS adaptation and connectivity control in heterogeneous environments with mobile receivers and static senders. By interacting with resource allocation controllers, MUSC allows the construction of QoS-aware distribution trees over networks with different QoS models and aims to keep sessions with an acceptable quality of experience in congestion periods. Furthermore, by interacting with mobility controllers, MUSC assures the session continuity with QoS and connectivity support. MUSC was evaluated in a simulation and in an experimental environment to analyze its convergence time as well as its efficiency in allowing seamless mobility and in keeping sessions with an acceptable quality level during handover.

(C) 2007 Elsevier B.V. All rights reserved.
\end{abstract}

Keywords: Multi-user session; Quality of Service; Mobility; Heterogeneous networks

\section{Introduction}

Next generation IP networks are expected to allow mobile users to access real-time group communication sessions over heterogeneous wireless environments with Quality of Service (QoS) support. Examples of these sessions are IPTV, video streaming and seismic activity reports, in which the session content is distributed to multiple mobile users at the same time (one source and multiple simultaneous receivers) [1]. Multi-user sessions can be classified as non-scalable and scalable, where the latter is composed by a set of flows with well-defined priorities, rates and QoS

\footnotetext{
* Corresponding author. Tel.: +351 239701226 .

E-mail addresses: ecoelho@dei.uc.pt (E. Cerqueira), 1mveloso@ dei.uc.pt (L. Veloso), augusto@dei.uc.pt (A. Neto), marilia@dei.uc.pt (M. Curado),edmundo@dei.uc.pt (E. Monteiro),pmendes@inescporto.pt (P. Mendes).

${ }^{1}$ Tel.: +35122 2094000 .

${ }^{2}$ Work developed under the affiliation of NTT DoCoMo Euro-Labs, Munich, Germany.
}

requirements as supported by common encoders, such as H.264, MPEG-2 and MPEG-4. In addition, the session distribution must be done in a multicast to save network resources, and the importance of each flow must be used to adapt the overall quality of the session to the availability of the different network traffic classes. This scheme allows the network to be independent from the encoders, which does not happen in transcoding approaches [2].

Fig. 1 illustrates a generic definition of a multi-user session as well as the distribution environment and the characteristics of the users. Additionally, it shows that a multi-user session is received by heterogeneous receivers according to the importance of each flow (from high to low).

The distribution of multi-user session content to multiple mobile subscribers with different devices and needs may be done using different QoS models (e.g., Differentiated Services or IEEE 802.11e), connectivity schemes (e.g., IP multicast or IP unicast) and access technologies (e.g., Worldwide Interoperability for Microwave Access - 


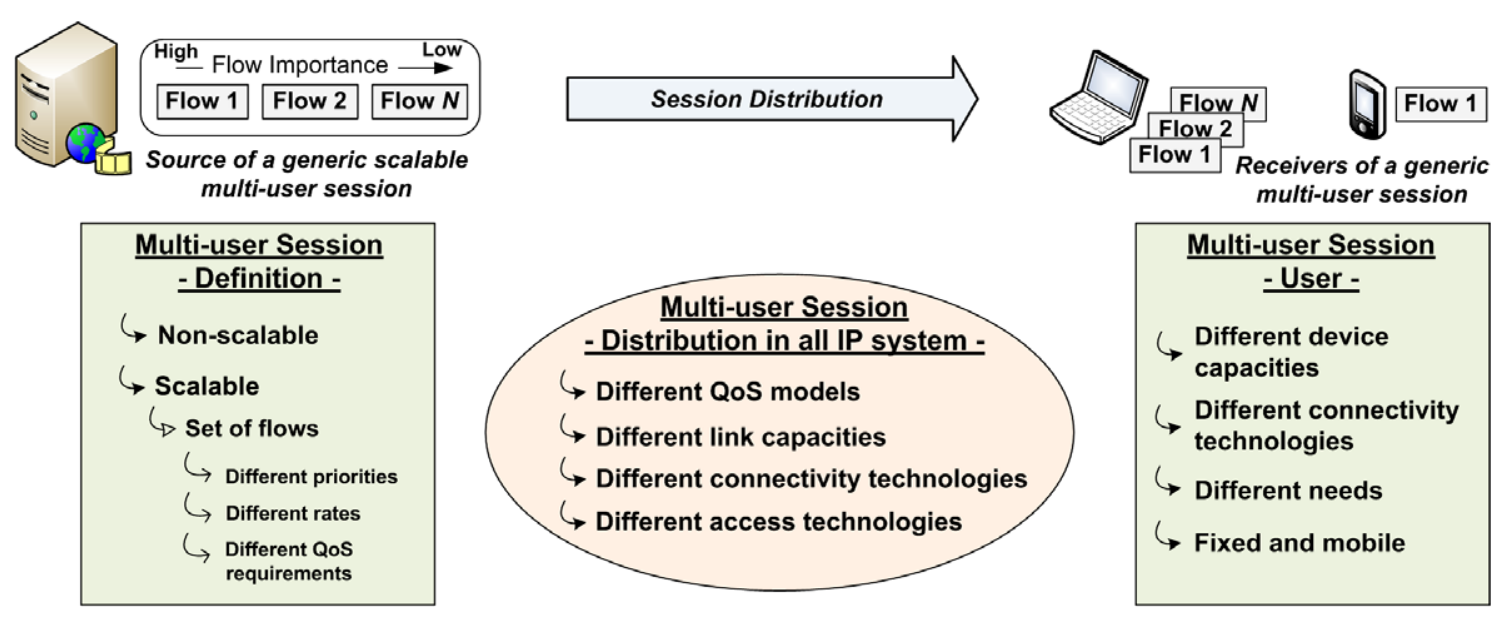

Fig. 1. Generic example of multi-user session definition and distribution.

WiMAX) [3]. In such heterogeneous environment the QoS guarantees, connectivity, ubiquitous access and mobility for multi-user sessions are requirements to the success of next generation wireless systems.

The distribution of multi-user sessions with end-to-end QoS support also requires the session QoS control in environments with asymmetric routing, which is not accomplished by most IP multicast protocols [4], such as Protocol Independent Multicast for Source-Specific Multicast (PIM-SSM). The above functionality requires the creation of distribution trees taking into account the QoS characteristics and current network conditions of the path from source to receivers. Moreover, due to the use of diverse QoS models in an all-IP system (or the same QoS model but configured with different traffic classes and performance metrics), flows of multi-user sessions need to be mapped into different service classes inside or between networks. However, current static approaches for QoS mapping or even guidelines for IP QoS mapping [5] alone are not sufficient to assure the quality level of sessions.

Furthermore, in order to increase the satisfaction of users and to avoid session blocking, it is necessary to keep multi-user sessions with acceptable quality level, independently of the existence of links with distinct capacities, movement of users or even due to a re-routing event caused by a failure in a network agent or link. For instance, in a congestion period, a QoS adaptation mechanism must be used to adapt the session to the current network conditions, by re-mapping the session to a different service class or controlling the session quality level by dropping and adding flows. In addition to the QoS control, a connectivity control scheme must be used to allow the end-to-end session continuity over networks that support the same and/ or different address realms, such as unicast to multicast and vice-versa.

In handover situations, the QoS and connectivity support for ongoing multi-user sessions must be done independently of hard or seamless mobility controllers. In terms of standard hard handover controllers, Mobile IP version 4 (MIPv4) [6], Session Initiation Protocol (SIP) [7] and Mul- ticast Remote-Subscription [8] can be pointed out. In order to improve the satisfaction of users, QoS support for seamless mobility controllers is also required, where packet loss and latency are reduced during handovers by using caching and buffering mechanisms.

This article describes the Multi-user Session Control (MUSC) [9] solution to manage the ubiquitous access and mobility for multi-user sessions across heterogeneous wireless networks. MUSC provides QoS mapping, QoS adaptation and connectivity control for ongoing multi-user sessions. From the mobility point of view, the interaction between MUSC and hard handover controllers allows the continuity of sessions with QoS support. The multi-user sessions controlled by MUSC can be supplied with seamless mobility capability through the communication with the Seamless Mobility of Users for Media Distribution Services (SEMUD) mechanism [10]. In addition, the creation of QoS-aware distribution trees associated with multi-user sessions is done based on an interface between MUSC and Multi-service Resource Allocation (MIRA) [11]. MUSC, SEMUD and MIRA were developed in the Quality of Service for Mobile, Multimedia, Multi-user Sessions (Q3M) project developed in cooperation between the University of Coimbra and NTT DoCoMo Euro-Labs.

The remainder of this article is organized as follows. Section 2 introduces the related work. A description of MUSC together with resource allocation and mobility controllers is presented in Section 3. Section 4 illustrates examples of the proposed solution. Section 5 presents evaluation results about the efficiency of MUSC in a simulation and in an experimental environment. Finally, conclusions and future work are summarized in Section 6.

\section{Related work}

The Real-Time Streaming Protocol (RTSP) [12] and SIP are IETF standard signaling protocols used to control the streaming and the access of users to announced sessions. SIP can also be used to control handover at the application layer, where it keeps the mobility support independently of 
the underlying wireless technologies and network layer elements. However, these end-to-end protocols are only suitable to control one-to-one or one-to-few single-user sessions supported by unicast environments and lack in terms of QoS and connectivity support.

In addition, existing mobility control proposals, such as MIPv4, MIPv6 [13], Hierarchical MIPv6 (HMIPv6) [14] and Fast Handovers for IPv6 (FMIPv6) [15], were developed to control the movement of unicast sessions. Concerning multicast, two mobility control methods can be pointed out: the bi-directional tunneling based on MIP, and the remote-subscription technique [16]. Both proposal lack QoS support and are dependent of specific connectivity technologies, such as multicast from source to home agent in the former, and the same end-to-end multicast address realm in the latter. Multicast mobility in IPv6 networks is also discussed by Schmidt and Waehlisch [17], where they present solutions for multicast handover only inside and between multicast-aware networks, avoiding its deployment in networks or backbones with non-multicast capabilities. In order to minimize the latency and packet losses, Context Transfer Protocol (CTXP) approaches together with anticipated handover schemes have been proposed to provide seamless mobility by allowing the setup of sessions in advance $[18,19]$. However, CTXP-based approaches alone are not sufficient to provide seamless mobility, since they do not setup sessions over heterogeneous environments.

Additionally to the mobility control, the heterogeneity of networks poses requirements for the mapping and adaptation of QoS, as well as for the translation among different connectivity schemes. Regarding connectivity control solutions, the end-to-end connectivity control over heterogeneous networks can be accomplished by using tunnelbased [20] or translation-based [21] approaches. The former requires the same IP multicast address realm in both access-networks and the latter allows the session connectivity mapping between unicast and multicast networks. Moreover, existing translation solutions provide unidirectional conversion between unicast and multicast realms [22]. However, the previous translation-based approaches do not provide the session connectivity control between multicast networks with different address realms, such as networks implementing SSM.

QoS mapping schemes are used to assure the mapping of sessions into network service classes. There are several proposals to map QoS into sessions; however they require proprietary modules on the end-systems and "expert" users to select the most suitable class [23], which reduce the system flexibility. Ruy et al. [24] proposed a centralized agent that classifies session requirements into service classes between networks with different QoS models. However, it is focused on the QoS metrics used by its agents and does not present the cooperation between agents to control QoS mapping along the end-to-end session path. Mammeri [25] proposed a mapping scheme that provides QoS control for unicast sessions across Integrated Services (IntServ) and Differenti- ated Services (DiffServ) models. However, this approach is dependent on the underlying QoS model.

In situations where it is not possible to assure the minimal QoS committed for a session or for some flows of that session, for instance, due to the unavailability of network resources, QoS adaptation solutions are used to adjust the overall quality of a session to the capability of different networks. However, most of the approaches require either the implementation of modules on the user side to join or leave multicast sessions [26] based on notification about the network conditions, or they need network devices to adapt the content coding (re-coding) to the available bandwidth [27]. The former only works in multicast-aware environments and, in the latter, networks are dependent of encoders, decreasing thus the system flexibility.

The coordination between network agents to provide session QoS mapping, QoS adaptation and connectivity procedures must be accomplished in a receiver-driven and source-initiated signaling approach. The Next Steps in Signaling (NSIS) IETF working group is developing a new signaling suite based on a two layer paradigm that aims to provide control information to network agents in unicast environments. The lower layer, called NSIS Transport Layer Protocol (NTLP), uses existing transport and security protocols under a common messaging layer, the General Internet Signaling Transport (GIST) [28]. The upper layer is an application-specific layer called NSIS Signaling Layer Protocol (NSLP). However, due to its single-user signaling nature, the NSIS must be extended to allow control operations for multi-user sessions over unicast and multicast networks.

The analysis of related work has shown that none of the approaches satisfy all requirements to control the access of multi-user sessions of fixed and mobile users with QoS mapping, QoS adaptation and connectivity support in heterogeneous networks. Most of the approaches were developed to be used in networks with specific QoS models or connectivity technologies. Hence, the University of Coimbra is working with DoCoMo Euro-Labs in a mechanism, called MUSC (Multi-User Session Control), described in this paper, intended to control the access and mobility of multi-user sessions in next generation wireless systems.

\section{Mobility management for multi-user sessions}

Mobility management for multi-user sessions over heterogeneous environments is achieved through the cooperation of MUSC with resource allocation and mobility controllers. Due to the heterogeneity of the networks, this approach is based on the separation of the session identifier and the network locator as proposed in the context of the NSIS framework. While the session identifier has a global meaning, the network locator is only relevant for the local network. Hence, each session is described in a Session Object (SOBJ) identified by a session identifier. Each session can also be composed by a set of flows, where the QoS parameters of each flow are described in the $Q o S$ 
Specification (QSPEC) object [29]. In addition, it is assumed that mobile receivers get, from the source, information about the available sessions, including the SOBJ and QSPECs. Each QSPEC includes the flow priority, bit rate, loss tolerance, delay and jitter.

The MUSC functionalities are implemented by MUSC agents, where a signaling protocol called MUSC-P, is used to coordinate QoS mapping, QoS adaptation, and connectivity mechanisms with other agents on the end-to-end session path. MUSC agents are developed in a modular and decentralized manner, where the former facilitates the inclusion of emerging technologies and the interaction with existing standards, while the decentralization permits a higher scalability. Agents are called access-agents when located in wired or wireless access-routers. Moreover, agents can have distinct roles in different edges for different sessions: in an edge router, an agent is called ingress-agent for sessions whose traffic is entering the network in that edge router, or egress-agent if the traffic is leaving the network.

\subsection{Components overview}

This section presents an overview of MUSC together with a description of resource allocation and mobility controllers.

\subsubsection{Mobility control}

An interface with mobility controllers allows MUSC to provide QoS and connectivity support also for ongoing sessions. The interaction between MUSC and MIPalike controllers, such as MIPv4, MIPv6 and FMIP, aims to fulfill the requirements of providing QoS control in mobile IP scenarios identified by the research community and reported in RFC 3583 [30], as well as it reduces signaling due to handovers as suggested in the NSIS working group [31]. Hence, MUSC assures QoS guarantees for an ongoing session in a foreign network, releases state associated with the session on the old path and controls the re-establishment of the session only from the nearest branch point to the mobile user and not end-to-end (from Home Agent (HA) in case of a MIP bi-directional tunneling approach or from the branch point nearest to the mobile user in case of a remote-subscription scheme). For instance, during a handover, MIP Home Agent (or previous access-router when FMIP is configured - IEEE 802.11 or IEEE 802.16 [32]) notifies MUSC about the movement of the user after receiving a corresponding binding process. After that, MUSC controls the session setup on the new path with QoS support and releases resources of the session on the old path. Furthermore, SIP-based handover can also be supported by the interaction of SIP and MUSC, allowing mobility support independently of the underlying wireless technologies. The reception of a SIP re-INVITE message in an access-agent allows MUSC to setup the ongoing session on the new path with QoS and connectivity support.
In order to control ongoing sessions in broadcast access networks, mobility controllers can also be extended to manage handover in networks with unidirectional links (e.g., Digital Video Broadcasting-Handheld, DVB-H) by interaction with the Link Layer Tunneling Mechanism (LLTM) [33] as explored by Miloucheva et al. [34]. LLTM adds a layer between the network interface and the routing scheme to emulate the full bidirectional connectivity. In this context, MUSC is notified about the movement of the user by the current mobility scheme, such as MIPv6. Based on this information, MUSC interacts with the local resource allocation controller to reserve resources on the downstream unidirectional channel and the upstream return channel taking into account the QoS characteristics of each path.

Additionally, SEMUD (Seamless Mobility of Users for Media Distribution Services) module enhances multi-user sessions controlled by MUSC with seamless mobility property. This improvement provides seamless intra and internetwork handovers through the combination of context transfer, caching and buffering mechanisms and an interface with mobility prediction schemes. Packet losses and delay are expected to be reduced by controlling the caches in access-agents and the buffers in mobile receivers. The packets associated with a multi-user session and received in the access-agent are stored in the cache and forwarded to the interested receivers. When those packets are received by a mobile device, they are stored in the buffer and consumed by the application. When a handover occurs, the data in the buffer of the mobile receiver will continue to be consumed by the application. When the handover is finished, the mobile receiver updates its buffer by fetching the missing packets from the cache in its new access-agent. The message sent to fetch the missing packets includes information concerning the available space in the buffer and the time stamp of the last packet received in the buffer before handover.

The use of context transfer and an interface with mobility prediction mechanisms allows SEMUD to notify MUSC about the mobility of users in advance (consequently, the setup of ongoing QoS-aware sessions is done before the handover). Different prediction schemes can be configured by network operators to inform SEMUD about the address of the next agent(s) and to start the seamless handover process. The decision on the SEMUD prediction approach depends on the business model used by the mobile provider and it is out of scope of this article. For instance, the SEMUD prediction interface can be configured to interact with Candidate Access Router Discovery (CARD) [35] to acquire information the access-agent candidate(s) for the handover. On the other hand, the use existing statistical prediction schemes [36] or complex pattern detection algorithms [37] also allow SEMUD to enhance the handover triggering. A comparison of mobility prediction algorithms to increase the handover trigger accuracy is explored by Sricharan et al. [38], and Michaelis et al. [39]. 
Upon receiving information about the candidate agent, the session context is transferred to it. SEMUD-P is a CTXP-based signaling protocol implemented to transfer the context of ongoing multi-user sessions between old and candidate access-agent(s). Additionally, information concerning the capabilities provided by the candidate access-agent(s) is collected by MUSC and conveyed by SEMUD-P to the current access-agent. At the old accessagent, the probed information concerning the available resources in the predicted access-agent(s), the signal-tonoise ratio and the knowledge regarding the access technologies, gives support to the handover decision. When the handover decision is made, the communication between SEMUD and MUSC allows the release of resources reserved on the old path, and on the new paths that the mobile device is not going to use (if no other users are subscribed to the same session).

\subsubsection{Resource allocation control}

Resource allocation functions are essential to manage bandwidth resources inside and between networks, by controlling the traffic load and by accepting or rejecting incoming sessions. This control can be performed in a centralized or decentralized mode by MIRA. In the centralized mode, MIRA is implemented in a central element acting as a QoS Broker scheme to provide admission control and resource reservation for sessions. This QoS entity also maintains information about the available service classes and current bandwidth condition of each class, allowing MUSC QoS mapping and QoS adaptation operations. However, in order to increase the system scalability, MIRA is configured (by default) in the decentralized mode by pushing the complexity of controlling the network resources to the edge MIRA agents and leaving the interior routers simple.

MIRA aims to build QoS-aware distribution trees associated with multi-user sessions in environments with asymmetric routing. In SSM-aware environments, MIRA provides support so that the multicast routing protocol, such as PIM-SSM, creates QoS-aware multicast trees taking into consideration the QoS characteristics and current bandwidth of the path from the sender to the receiver. This is done through updating the Multicast Routing Information Base (MRIB) with the QoS reserved-path during the resource allocation work, enabling PIM-SSM to create the QoS-aware trees in environments with asymmetric routes. QoS management for unidirectional links is being considered to provide resource reservation for the downstream (unidirectional) and upstream (emulated return channel using wireless access networks) links also taking into account the QoS characteristics of each path.

In wireless networks, MIRA interacts with IEEE 802.11e, IEEE 802.16-2004 [40] and IEEE 802.16e-2005 [41] (IEEE 802.16-2004 and IEEE 802.16e-2005 will be called IEEE 802.16 in the article) Medium Access Control (MAC) elements to control resources for service classes in wireless links. For instance, in IEEE 802.11e networks, a MIRA agent is also implemented in the QoS-Access Point (QAP) [42]. In this example, DiffServ classes are assumed in the wired link from the access-agent to the access-point and IEEE 802.11e classes from the access-point to the mobile device. At the system bootstrap, MIRA (located in the access-agent) collects the available service classes and resources capability of each class from the agent placed in the QAP. During the session setup, MUSC, in the access-agent, queries MIRA about the service classes in wired and wireless interfaces. After receiving the requested information, MUSC selects the wired and wireless QoS classes to be used for the session and triggers MIRA to accomplish the resource reservation process. Hence, MIRA performs the reservation in the wired interface (by configuring a DiffServ class) and signals the MIRA agent in the QAP to resume the resource reservation in the wireless link. In the QAP agent, MIRA composes a Traffic Specification (TSPEC) and triggers the Hybrid Coordinator (HC) element with an ADDTS (add Traffic Stream) request to allow the resource reservation in the selected wireless class. A similar process is performed to provide QoS support for sessions in a WiMAX system, where MIRA agents are collocated in Connectivity Service Network (CSN) and Access Service Network-Gateway (ASN-GW) entities and interact with the IEEE 802.16 MAC layer to request the creation of QoS-aware service flows associated with a multi-user session.

In multicast-aware networks, MIRA follows the SSM model to control multi-user distribution and it allocates a SSM channel (e.g., source and multicast group) to be used by each flow of a session. In unicast-aware networks, a pair of IP unicast addresses and transport ports is allocated to identify each flow of a session. Based on the information regarding the distribution channel identifiers provided by MIRA, MUSC controls the session connectivity independently of the address realm implemented along the session path.

In addition, MIRA uses an interface to exchange information with unicast routing mechanisms, such as Open Shortest Path First (OSPF) [43] and Border Gateway Protocol (BGP) [44], to retrieve information on the network interface towards a user or another agent by querying the routing table. The local routing scheme can be configured by the network provider based on shortest path approach or based on other criterions such as load balance, delay or/and bandwidth, which are usually used by QoS routing approaches [45]. The combination of resource reservation and QoS routing allows a fine control over the routes and resources, while increasing the complexity of the network due to the increment of computational efforts and state needed to maintain information about the available routes. In order to contribute to the system robustness, MIRA detects re-routing events by intercepting router advertisements generated by the local routing protocol when an interface goes down or comes up (e.g., router Link State Advertisement (LSA) used by OSPF). For example, the communication between MIRA and OSPF allows the 
detection of re-routing events inside a network, while internetwork re-routing changes are detected through the interaction with BGP. After detecting a re-routing event, MIRA tries to accomplish a faster re-establishment of the affected sessions on the new path without damaging the already current sessions. When the new path is overloaded, MUSC is requested to adapt the sessions to the current network conditions. This procedure aims to avoid session blocking and to keep sessions with acceptable quality.

The MIRA Protocol (MIRA-P) is used to exchange control information between MIRA agents. MIRA-P is being specified based on NSIS NSLP, where it controls the state maintenance by soft-state. Between networks, MIRA performs its functions based on Service Level Specification (SLS) [46].

\subsubsection{Multi-User Session Control}

MUSC manages the mobility of multi-user sessions, as well as the ubiquitous access of users to those sessions. The cooperation between MUSC agents allows QoS mapping, QoS adaptation and connectivity of multi-user sessions along a heterogeneous end-to-end path. The ubiquitous access control of a fixed user is done in the access-agent through an interface with SIP, while the continuity of an ongoing session is guaranteed through an interface with mobility controllers, such as SEMUD and MIP.

In ingress and egress-agents, the QoS mapping mechanism performs a dynamic mapping of the session requirements into the available service classes. The QoS mapping mechanism compares, one by one, the QoS parameters requested for each flow of the session and the list of available classes. Then, it maps each flow into the suitable network classes, based on three methods: perfect, sub-perfect and hybrid matches. The perfect match is the preferential method, since it supports the full QoS requirements and bandwidth committed for all flows of a session. When the preferred class does not have enough bandwidth to assure the minimal rate of the session, the QoS adaptation mechanism is triggered, which then may decide to try a sub-perfect or a hybrid mapping. The sub-perfect match maps all flows of a session to a service class that supports QoS parameters different from the ones described in the QSPEC. This method aims to avoid session blocking and re-ordering of packets. The hybrid match assures the allocation of, at least, the high priority flows of a session to the preferred class. The remainder flows are mapped to a less significant class. It can be used when the packet re-ordering is not crucial. For instance, it can be suitable for scheduled video and audio, where it is more important to ensure an intelligible audio flow than a perfect video.

When the mapping process is not optimal, for instance, due to a selection of an overloaded service class, the adaptation mechanism is triggered. The adaptation mechanism operates based on the QSPEC and the current network conditions by interacting with MIRA. The three adaptation methods are as follows. The first method drops/adds low priority flows of a multi-user session. Therefore, when the maximum bandwidth of the preferred class cannot assure the QoS committed for a low priority flow, this flow is removed from the outgoing interface and classified into the sleeping state by MUSC. Sleeping flows are awoken when the network capability becomes available again and the session full rate is supported. On the other hand, the re-mapping adaptation method requests the mapping of the session to another class (using the sub-perfect or hybrid mapping). The last method, called service class re-adjustment, can be used to try the accommodation of the session into the preferred class, by requesting the re-adjustment of the maximum (extra) bandwidth assigned for the service classes to the resource allocation controller.

If the ingress or egress-agent is in the frontier between unicast and/or multicast address realms, MUSC configures its connectivity translator, allowing multi-user sessions to be distributed independently of underlying connectivity technology. In unicast-aware networks, the packet distribution is handled in a multicasting way to save network resources. The packet duplication is performed near to the receivers (application layer multicasting). The end-toend coordination of a chain of connectivity translators supports any layout of heterogeneous networks and end-hosts.

A receiver-driven and source-initiated protocol, called MUSC-P, is used to coordinate QoS mapping, QoS adaptation and connectivity control mechanisms with other edge-agents along the end-to-end session path based on a soft-state approach. MUSC-P is compliant with the NSIS framework, in which it can be included as an extra NSIS NSLP. MUSC-P operates in a receiver-driven approach, since it is triggered at the access-agent (agent located in wired or wireless access-router). It is source-initiated since MUSC starts the QoS and connectivity configuration of its agents at the agent nearest to the source, or at the first agent in the path towards the source that contains the requested session. When MUSC is triggered by MIP (or alike) mechanisms, only source-initiated functions are done to control the quality level of sessions from the home agent (source in this case) to the moving receivers.

\subsection{Communication interfaces}

This section describes the system functional interactions between MUSC, MIRA, SEMUD and the interfaces used for communication between these modules and existing standard solutions such as SIP, Session Description Protocol (SDP) [47], PIM-SSM, OSPF, BGP, Internet Group Management Protocol (IGMPv3) [48], Multicast Listener Discovery (MLDv2) [49], MIP-based controllers, DiffServ elements and Layer 2 wireless controllers. The location, functional interactions and interfaces of the proposed system are illustrated in Fig. 2.

The MUSC-SIP interface allows users to access or leave a multi-user session ubiquitously. Applications compose a SIP/SDP message to join announced sessions. This message is received by a SIP-proxy in the access-network, 


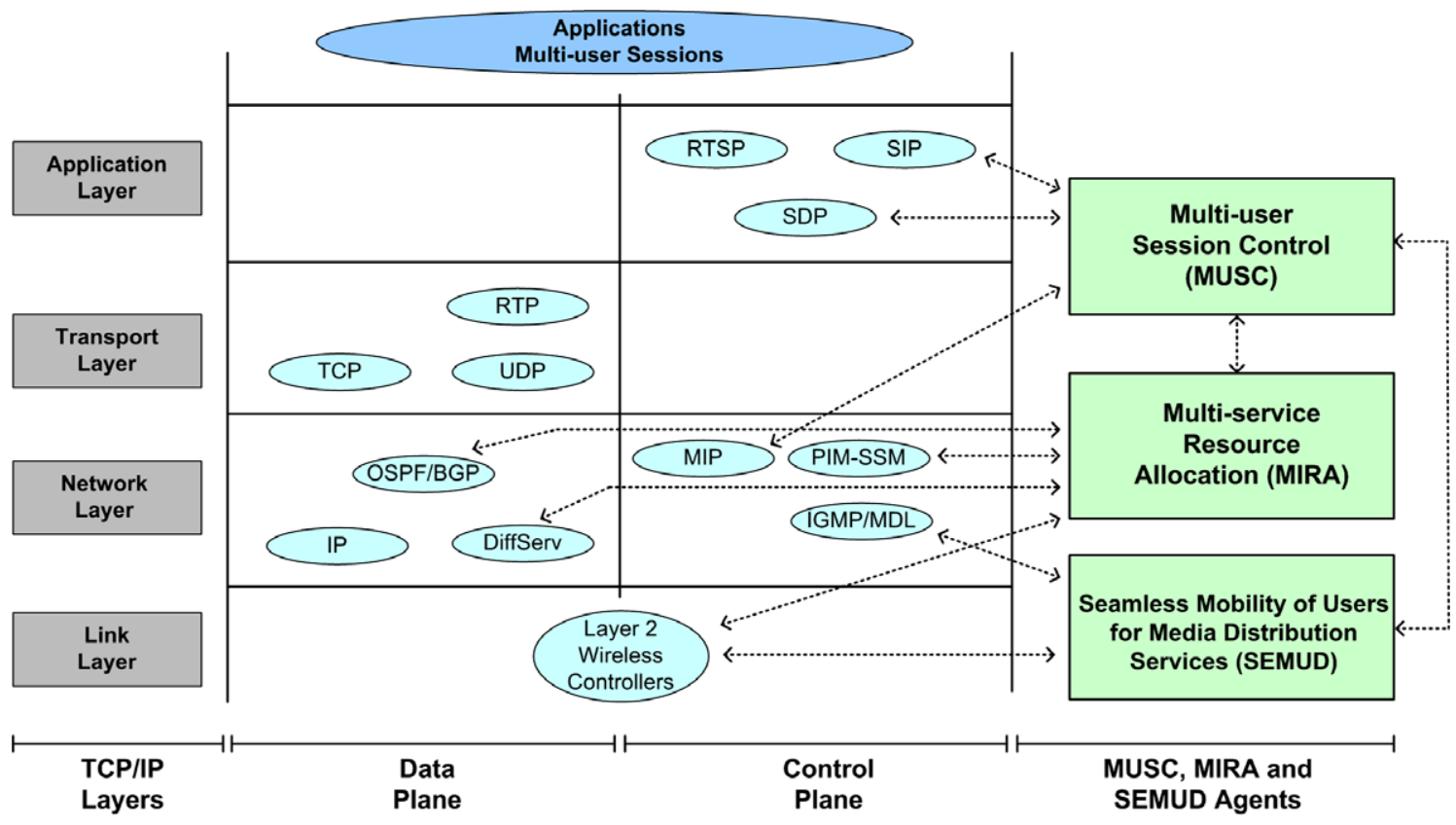

Fig. 2. Relationship of the components in a TCP/IP stack view.

which can accomplish Authentication, Authorization and Accounting procedures (AAA) as supported by the IETF Diameter solution, together with AAA servers [50]. An example of the interaction between SIP and Diameter to provide AAA control is when a SIP-server receives a message, such as SIP INVITE, SIP re-INVITE or SIP REGIS$T E R$, and interacts with the Diameter-client for the request to the AAA server [51].

The Diameter-client contacts its server to determine if the user is allowed to receive the required session. Based on information about the user's credentials, such as session identifier, username and password, the Diameter-server starts the user access control process. If the AAA process succeeds, the user is authorized to access the required content and a reply message is sent to the Diameter-client. Otherwise, an access reject message is sent. After receiving a response message, the Diameter-client notifies the SIPserver about the status of the AAA process (accepted or rejected). If the access is rejected, a SIP message is sent to the receiver with a rejection code. If the access is accepted, the SIP-server forwards the session information to the receiver's access-agent. The reception of this information activates the MUSC access control. After the conclusion of the MUSC operations, the application is informed, through a SIP/SDP message, about the acceptance status of its request. For multicast-aware devices placed in multicast-aware access-networks, MUSC also informs the receiver about the multicast channel to be used for each flow of a session (consequently, IGMPv3 or MLDv2 messages are sent to the access-router). SIP-based handover can also be supported by the interaction of SIP and MUSC. The reception of a SIP re-INVITE message in an access-agent also allows MUSC to setup the ongoing session on the new path with QoS and connectivity support.

The MUSC-MIP interface allows continuity of QoSaware ongoing sessions over heterogeneous networks when mobility is managed by MIP-alike controllers, such as MIPv4, MIPv6 and FMIP. MUSC assures QoS guarantees for ongoing session in a foreign network and controls the release of resources on the old path. It is assumed that the AAA control of the user in a visited network or in a new access-agent in the network is done through the interaction between MIP-alike and Diameter agents [52] in a similar manner as explained before.

The MUSC-SEMUD interface allows seamless mobility for multi-user sessions. When a new session is accepted by MUSC, SEMUD is triggered to create a cache for the session (if the session does not exist) and to receive the SOBJ. During a handover, SEMUD transfers the session context, requests AAA functions (as explained before) and triggers MUSC in predicted access-agent(s) to setup the session and to collect information concerning the capability and connectivity of the new paths. After handover, the information about the connectivity capability in new multicast accessnetworks is used by SEMUD, in the mobile node, to join the multicast channels associated with the flows of a session in the new access-agent, by triggering an IGMPv3 or MLDv2 join message.

The MUSC-MIRA interface is implemented in all edge agents providing end-to-end connectivity and QoS support over heterogeneous networks. MUSC triggers MIRA by querying it about the QoS characteristic of the classes inside or between networks and requesting the allocation of each flow of a session into a selected class. As a response, MUSC gets from MIRA information about the unicast 
flows or multicast trees to which the flows of a session were associated. MUSC also triggers MIRA to release the resources of unsubscribed sessions (e.g., due to handover).

The MIRA-DiffServ interface is used by MIRA to collect information about the network services (loss, delay, jitter and available bandwidth) by checking the Per-HopBehavior (PHB) table on each network node from the ingress to the egress-agent. During a resource reservation process, MIRA adjusts the bandwidth share of the PHB of the selected network service in each router of the network ingress-egress path.

The MIRA-Layer2WirelessController interface is used by MIRA to collect information on the wireless class capabilities and to interact with MAC layer to configure the MAC classifier according to the class selected by MUSC.

The MIRA-PIM-SSM interface allows the former to update the MRIB on each route and activates the PIMSSM to create and release multicast channels associated with a multi-user session.

The MIRA-OSPF/BGP interface allows MIRA to retrieve information about the outgoing interface to reach a user or agent for a certain resource allocation request and to acquire information about re-routing events when a network interface becomes down or up.

\subsection{Signaling associated with handovers}

MUSC-P messages were specified in accordance with the NSIS framework, where MUSC uses the service offered by GIST to transport the messages between agents. Four mes- sage types are used to exchange control information between MUSC agents, namely, the SessRequest, SessResponse, SessAnnounce and SessRefresh messages. The SessRequest message is sent, with the IP router alert option, by a MUSC access-agent towards the agent where the requested session is activated. The destination of the SessRequest message is the agent closest to the source of the signaled session. However, this message can be stopped by the first agent discovered in the path towards the source with the requested session (i.e., the agent that has another branch point for the session). The SessResponse message is used to setup the session from the agent in which the session is found towards the MUSC access-agent (or HA in case of MIP), where the IP address of each downstream agent is provided by MIRA. In addition, SessAnnounce messages are sent periodically by a MUSC ingress-agent to other agents in the same network. These messages are useful to announce the sessions currently activated in an ingress-agent, decreasing MUSC-P overhead in multihomed networks, by keeping signaling local. Moreover, SessRefresh messages are sent periodically by downstream MUSC agents to upstream agents that are the ingress point of the sessions to be refreshed. If the state of a session is not refreshed in a certain period of time by using SessRefresh messages, its state is removed by soft-state.

Fig. 3 presents an example of the message sequence used by SEMUD-P, MUSC-P and MIRA-P to setup a session in advance due to inter-network mobility. In this example, the mobile node and the access-networks are assumed to be SSM multicast-aware. It only shows the message sequence

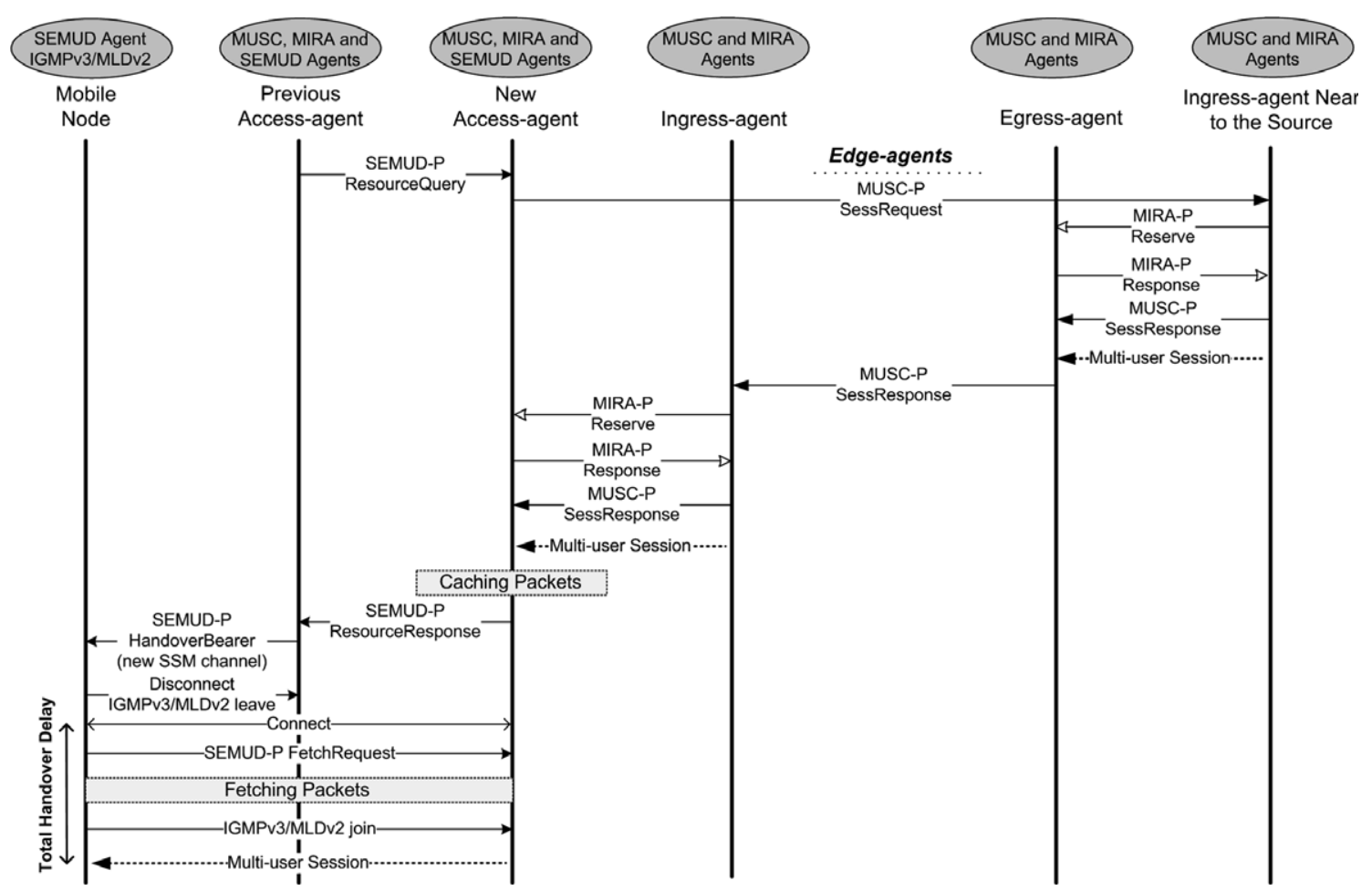

Fig. 3. Message sequence used by SEMUD-P, MUSC-P and MIRA-P in an inter-network handover. 
in the access-networks and in the network to which the source is linked. In addition to MUSC-P messages, four SEMUD-P messages, called ResourceQuery, ResourceResponse, HandoverBearer and FetchRequest, and two MIRA-P messages named Reserve and Response are associated with the handover. A SEMUD-P ResourceQuery message is used to transfer the session context to the predicted agent(s). Due to the SEMUD request, MUSC is triggered in the new access-agent to setup the ongoing session on the new path. Hence a MUSC-P SessRequest message is sent toward the source and captured by the agent near to the source. In order to reserve QoS resources in the network service selected by MUSC, a MIRA-P Reserve message is sent to notify MIRA agents (core and edge routers) along downstream about the amount of resources required for each ongoing flow. Moreover, an upstream MIRA-P Response message is triggered in the egress-agent or access-agent to inform the ingress-agent about the result of the requested operation (admission control - accepted or not).

When all ingress procedures are performed by MIRA and MUSC, a MUSC-P SessResponse message is sent to the next downstream agent towards the access-agent of the receiver. The SessResponse message stops when it reaches the access-agent of the receiver. Afterwards, a SEMUD-P ResourceResponse message is sent to inform the previous access-agent. The reception of such message allows SEMUD to take the handover decision and to send a Handover Bearer message informing the IP address of the future access-agent and the new distribution trees (multicast channels) allocated to each flow of the session. Before disconnecting from the old access-agent, SEMUD informs MUSC that a receiver will detach from this access-agent, allowing MUSC to control the number of users associated with the session. In case of failure of the mobile node or its wireless link, the MUSC agent can still acknowledge this situation by means of the information collected from IGMPv3/MLDv2 periodic queries. Upon the attachment of the receiver to the new access-agent, a FetchRequest message is sent from the mobile node to request the nonreceived packets associated with the session and stored in the cache. After the reception of missing packets IGMPv3/MLDv2 is triggered to join the new multicast channels. This sequence avoids extra intelligence and processing in the mobile node to perform the re-ordering of packets associated with flows of a session coming simultaneously from the unicast and the multicast interfaces.

In order to present the MUSC behavior due to a hard handover scenario, Fig. 4 shows an example of message sequence used by MIPv4, MUSC-P and MIRA-P to provide the continuity of an ongoing session with QoS support. It is assumed that the mobility is controlled by a

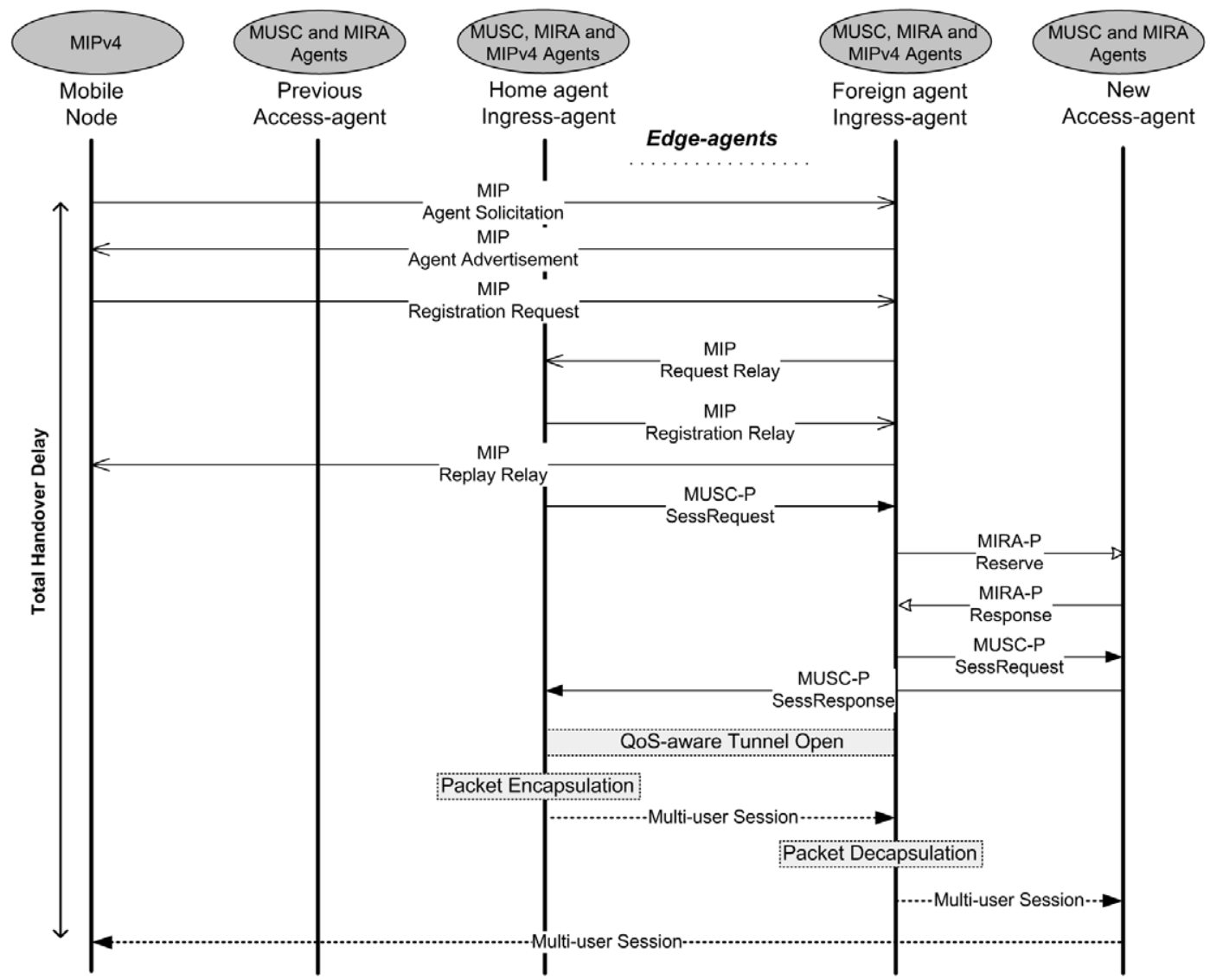

Fig. 4. Message sequence used by MIPv4, MUSC-P and MIRA-P in an inter-network handover. 
MIP bi-directional tunneling approach, where MUSC agents are collocated with MIRA at the edges of the networks. Furthermore, HA and Foreign Agent (FA) are also placed in the unique ingress point of access networks. Since handover controllers are not the focus of this article, the use of MIPv4 is assumed in Fig. 4. If MIPv6 would be supported, the FA would not be required on the data path. The same scenario would also be used with a FMIP solution to control the mobility of users.

The handover process starts when the mobile node sends a MIP Agent Solicitation message and receives a MIP Agent Advertisement message to determine whether it is on its home network or on a foreign network. These messages are not used if HA and FA are configured to advertise their presence via agent advertisement messages. Upon moving to a foreign access-network and obtaining a careof-address of the foreign agent, the mobile node registers its new address with its home agent through the exchange of MIP Registration Request and Registration Reply messages possibly via the foreign agent. After the registration process, the MIP HA notifies MUSC to control the session quality level on the new path (which includes the QoSaware tunnel between the HA and FA). Based on the session identifier associated with the moving node and supplied by the HA, MUSC retrieves the correspondent QSPEC object stored in the ingress agent. After that, the interaction between MUSC and MIRA allows the creation of a QoS-aware path to be used for the session also along the path from the home agent to the mobile node. All packets sent to the mobile node's home address are intercepted by its home agent, tunneled by the home agent to the mobile node's care-of-address, received by the foreign agent, and finally delivered to the mobile node with QoS support.

\section{Examples of the MUSC functionality}

This section presents three examples of the MUSC functionality. The first example shows an overview of end-toend procedures to allow a fixed receiver to access a multiuser session. The second example describes the operations to provide seamless mobility for receivers with QoS and connectivity control. Finally, the third example is focused on MUSC QoS mapping and QoS adaptation procedures due to an inter-network handover controlled by MIP.

\subsection{Session access control}

Fig. 5 shows how MUSC operates to allow a receiver to access a multi-user session over heterogeneous environments, by controlling the end-to-end session setup. Upon receiving the session announcement, the application on $\mathrm{R} 1$ uses a SIP/SDPINVITE message to subscribe the session Sl(i). The SIP-proxy forwards the message to the access-agent connected to $\mathrm{R} 1$ based on the previous registration and AAA control. Afterwards, the MUSC-P is triggered in access-agent N.1.3 to send a SessRequest message towards the source (ii). This message is received by the agent closest to the source, because the session is not present in any agent along the signaled path towards the source. In the ingress-agent N.2.1, MUSC triggers MIRA to query information about network services in the network path towards the access-agent N.1.3. In this case, the QoS characteristics of the path between the ingressagent N.2.1 and egress-agent N.2.3 are provided, because the latter is the exit point for the session in $N 2$. Based on the response and QoS parameters described in the QSPEC object, MUSC selects the appropriate network service and

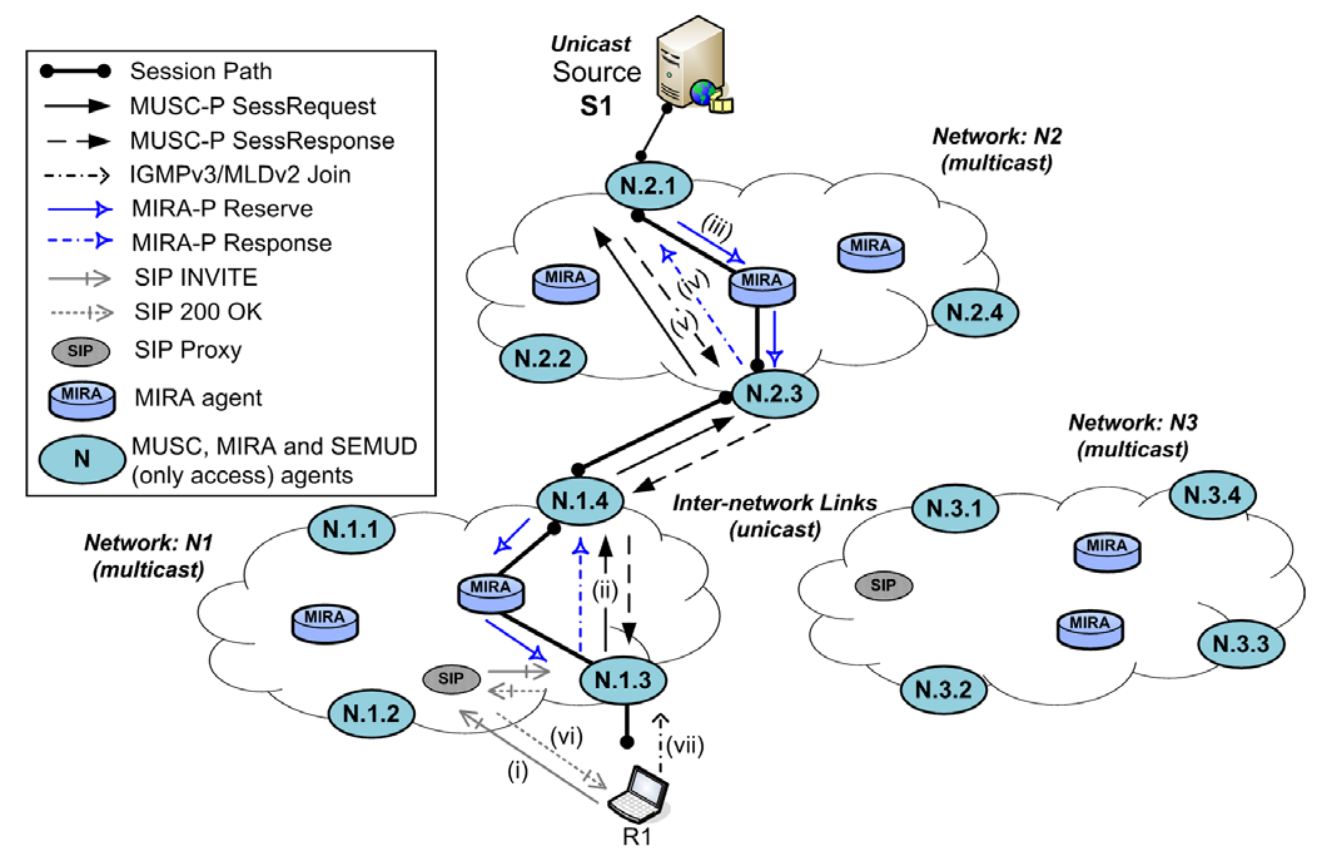

Fig. 5. Session access control. 
requests MIRA to configure the required bandwidth based on the perfect match method.

After admission control, MIRA updates the resources of the requested network class and configures the multicast (configuring the MRIB) in the path towards the egressagent N.2.3 by using a MIRA-P Reserve message (iii). Upon receiving a MIRA-P Response message (iv), MIRA concludes its intra-network operations and notifies MUSC about the multicast SSM channels allocated for each flow of the session. This information allows MUSC to start translating all incoming flows associated with the session to the indicated intra-network multicast trees.

After the operations in the ingress-agent N.2.1, MUSC in the egress-agent N.2.3 is triggered by a MUSC-P SessResponse message (v). This message allows MUSC to configure its translation state with information about the SSM channels to be used for each flow in the multicast network N2. The interaction with MIRA occurs as described before. Since the agent N.2.3 is an egress-agent, PIM-SSM is triggered by MIRA to create multicast trees for each flow of the session in the QoS reserved-path. Moreover, MIRA collects QoS information by checking the inter-network SLSs. Since unicast is used between networks, MIRA acquires from the SLS the unicast IP address of the next agent (in this case, agent N.1.4) as well as a pool of available ports. This unicast connectivity information allows MUSC to distribute sessions in a multicasting manner, where it maps all packets coming from the intra-network to the pair of source and destination IP addresses and ports allocated for each flow between $N 2$ and $N 1$.
After all edge operations in the downstream path, the MUSC-P SessResponse message reaches the access-agent N.1.3, allowing MUSC to update its connectivity translation state. Consequently, MIRA is required to control the multicast tree creation inside $N 1$ and to inform the available service classes on the wireless interface. After MUSC QoS and connectivity control operations, MIRA is triggered to configure the required bandwidth in the selected wireless service class. In addition, MUSC notifies SEMUD to activate the cache for the session and to receive information concerning session-context (SOBJ). Finally, MUSC sends a successful SIP 200 OK message to the receiver R1 (vi). In a multicast access-network, MUSC includes information about the SSM channels allocated for the session in N1 in the SIP response message. It is used for the application on the receiver to join multicast channels by using an IGMPv3 or MLDv2 message (vii). If unicast is used, MUSC maps each flow of $S 1$ to a list of interested users, and performs the required packet duplication only in the access-agent, saving thus network resources.

\subsection{Seamless handover control}

Fig. 6 assumes the existence of an anticipated prediction scheme that interacts with SEMUD to notify the IP address of the predicted access-agent. Based on the predicted information, SEMUD verifies that R1 (step a) is moving away from the access-agent N.1.3 to the candidate access-agent N.3.2. Upon receiving the IP address of the predicted access-agent, SEMUD-P sends a ResourceQuery message to the SEMUD agent in the access N.3.2 (i).

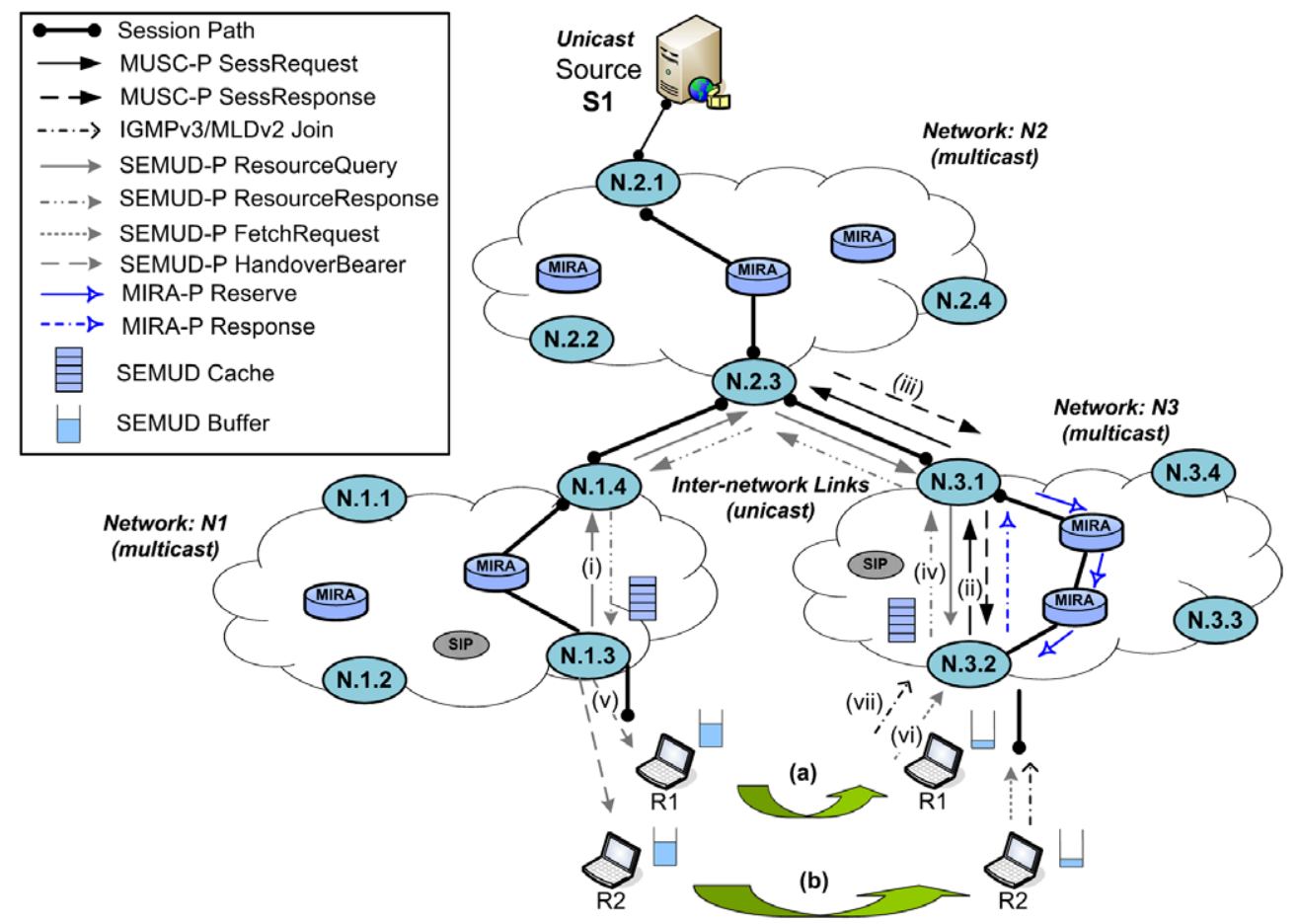

Fig. 6. Seamless handover management. 
After being triggered by SEMUD, MUSC verifies that $S 1$ is neither locally active nor in N3 and a MUSC-P SessRequest message is sent towards the source of the session (ii). This message is stopped in agent N.2.3, since it has another branch with the same requested flows of $S 1$. In this agent, the interaction among MUSC and MIRA provides QoS and connectivity control between N.2.3 and N.3.1. Afterwards, a MUSC-P SessResponse message is sent (iii), allowing QoS and connectivity functions in all agents along the downstream path.

Following all MUSC and MIRA operations in accessagent N.3.2, SEMUD is triggered by MUSC to activate the cache for the session and to receive information concerning session-context (including the new SSM channels used for each flow in N3). Hence, a SEMUD-P ResourceResponse message is sent to the SEMUD agent in N.1.3 (iv), allowing it to take an handover decision based on the information regarding the available resources in the predicted access-agent and the signal-to-noise ratio. In the access-agent N.1.3, SEMUD sends a HandoverBearer message (v) to inform R1 about the IP address of the future access-agent and the SSM channel allocated to each flow. After the handover, SEMUD informs MUSC to adjust the number of receivers associated with $S 1$ in the previous agent.

During handover, the packets are stored in the cache of the new agent and after the attachment of R1 to that agent, SEMUD-P is triggered to send a FetchRequest message (vi) to recover missing packets and to synchronize the packet reception with the cache in agent N.3.2. The recovered packets are sent from the cache to the buffer via unicast connections. This requires encapsula- tion of the multicast packets into unicast packets, which SEMUD in R1 de-encapsulates before putting them in the session buffer. This functionality avoids packet replication to other receivers subscribed in the same multicast group and also attached to agent N.3.2. After receiving all the fetched packets, SEMUD triggers the IGMPv3/ MLDv2 to join the multicast channel allocated for each flow of the session.

After the handover of $\mathrm{R} 1, \mathrm{R} 2$ is also moving (step b) and SEMUD triggers MUSC to pre-set the session on the new path. Upon receiving the SOBJ transferred by the SEMUD-P ResourceQuery message, MUSC access control verifies that $S 1$ is already presented in the agent N.3.2, increments the number of receivers receiving the requested session and requests MIRA to configure the required resources in the wireless link. After the MUSC reply, SEMUD associates R2 with the existing cache and sends a ResourceResponse message to the previous agent to complete the handover, which is accomplished as explained for R1. However, MUSC in access-agent N.1.3 releases the state associated with $S 1$, triggers SEMUD to remove the cache and MIRA to erase network resources and multicast trees of $S 1$ in N1. In the agent N.1.4 and agent N.2.3, MUSC removes the $S 1$ state (by interacting with MIRA), because no MUSC-P SessRefresh message arrives to these agents before the expiration of the MUSC clean-up interval.

\subsection{Hard handover control}

This example shows MUSC procedures to accomplish QoS mapping and adaptation for multi-user sessions due

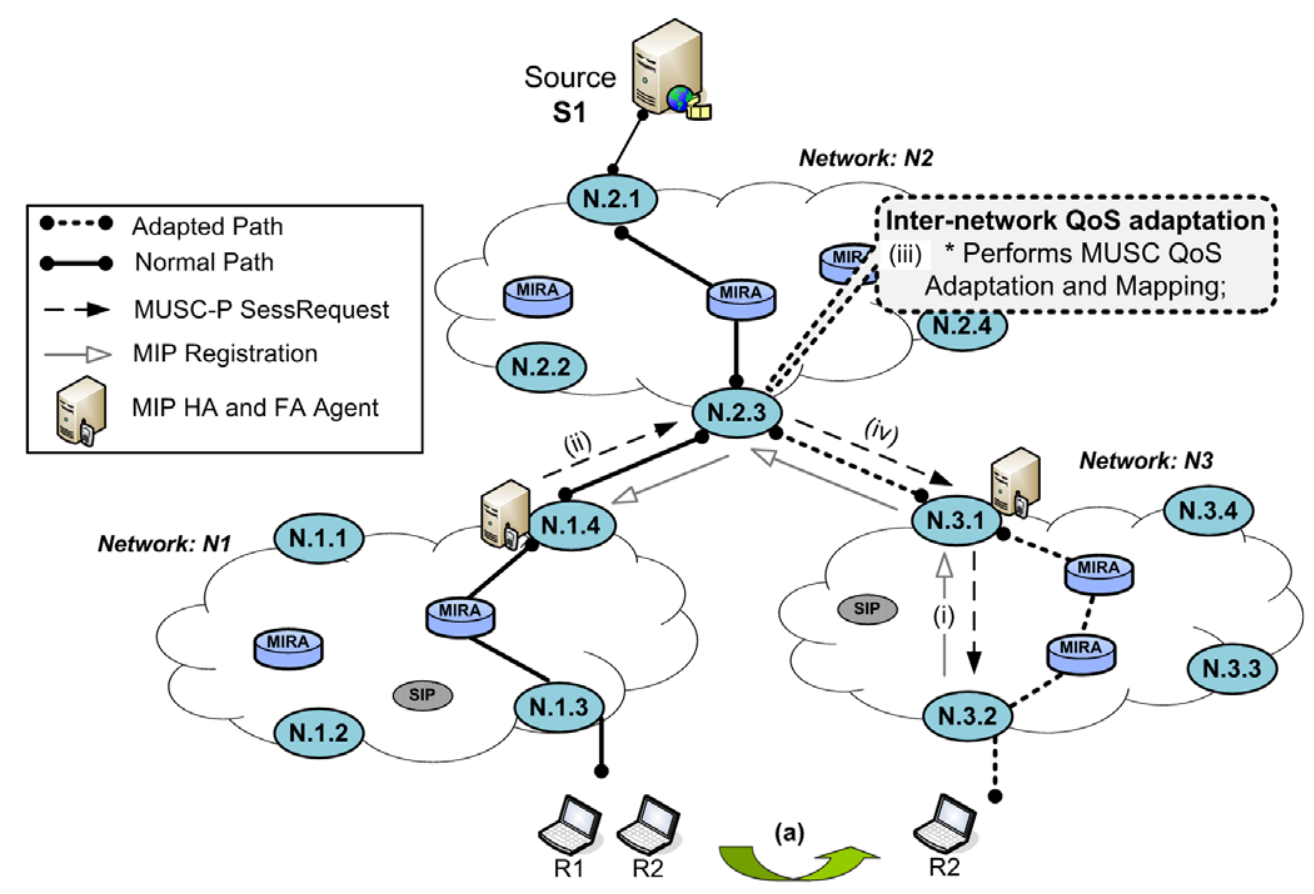

Fig. 7. Hard handover control with QoS adaptation support. 
to an inter-network handover. The mobility is controlled by a MIP bi-directional tunneling approach, where MUSC agents are collocated with MIRA at the edges of the networks. Additionally, MIP HA and FA agents are also placed in the unique ingress point of access networks. The scenario in Fig. 7 has three networks with different QoS models and $S 1$ is being sent to R1 and R2. When $\mathrm{R} 2$ moves to the agent N.3.2, it receives a router advertisement message and acquires a care-of-address of the foreign network. After that, R2 registers its new address with its HA through the exchange of registration messages (i). Upon finishing the registration process, the HA placed in N1 notifies the MUSC agent N.1.4 to control the session quality level on the path towards N.3.2 (which includes QoS-aware tunnels between HA and FA). Based on the session identifier associated with R2 and supplied by the HA, MUSC in agent N.1.4 consults its state and retrieves the QSPEC object. After that, MUSC triggers MIRA to query information on the available classes and their QoS characteristics of the inter-network link between N1 and N2.

Based on the MIRA response and on the QoS parameters collected in the QSPEC object, the MUSC mapping mechanism compares, one by one, the QSPEC object parameters of each flow with the capability of each class. After a successful match, it selects the most suitable service class for each flow. According to the priority of each flow, MIRA is triggered to configure the required bandwidth in the preferred class (inter-network path from N.1.4 to N.2.3). After the resource reservation process, MUSC is triggered and controls the quality level of the session on the remainder path. Hence, a SessRequest message is sent to the agent N.2.3, which verifies the QSPEC object of each flow and interacts with MIRA in the same way as explained before.

During the interaction of MUSC and MIRA for the configuration of a service class for the ongoing session between $N 2$ and N3, MUSC is notified since the preferred service class has not enough resources to accommodate the less priority flow of $S 1$. Since MUSC supports flexible methods to control the session quality level, it can be configured by operators with different profiles. For instance, based on the local configuration, MUSC uses a combination of the Re-mapping adaptation and Hybrid Match mapping methods to avoid the session blocking. Thus, MUSC requests the allocation of the highest priority flows into the preferred network class while the remainder flows are mapped to a less important class. After all the QoS control operations performed by MUSC and MIRA in the agent N.2.3, MUSC-P is triggered to signal the remainder downstream agents. All agents along the downstream adapted path will accomplish the same QoS control operations, as explained before, in order to resume the creation of a QoS-aware path for the ongoing session. In agent N.3.2, MUSC selects the preferred class for the session based on the available wireless classes (e.g., IEEE 802.11e or IEEE 802.16) and resources.

\section{Performance evaluation}

Performance evaluation of the MUSC proposal was carried out based on simulation and prototype experiments. The objective of the simulation experiments was to analyze the efficiency of MUSC in controlling the access of users to a session compared with an only SIP-based approach, by verifying the convergence time and signaling overhead during the session setup. MUSC is simulated together with MIRA and SEMUD, where the convergence time, the benefits of the buffering and caching mechanisms in reducing the packet lost due to handovers are analyzed. The objective of the prototype experiments was to verify the impact of MUSC QoS mapping and QoS adaptation procedures in controlling the quality level of a session due to an inter-network hard handover managed by MIP. This was done by measuring the throughput and the Round Trip Time (RTT) observed by receivers in the home and foreign networks.

\subsection{Simulation experiments}

The simulation was done using the Network Simulator 2 (NS2) and for each set of experiments, random topologies with twenty routers (four edges and sixteen interiors) were generated by BRITE (Boston University Representative Internet Topology Generator). The intra and inter-networks links have a bandwidth of $100 \mathrm{Mb} / \mathrm{s}$ and their propagation delay was assigned according to the distance between the edges of each link. Receivers are connected to IEEE $802.11 \mathrm{e}$ wireless links with a bandwidth of $11 \mathrm{Mb} / \mathrm{s}$. A SIP Digest authentication scheme was also supported by the SIP-proxies to provide authentication for the users during the session setup process based on a credential composed by username and password. During handover, the user's credential is transferred by SEMUD and the reauthentication process was performed in the foreign access-network.

\subsubsection{Session access control for MUSC and SIP}

This simulation experiments compares the convergence time and signaling overhead of MUSC and SIP to allow several users to access a published session. Moreover, the impact of using MUSC and MIRA to provide connectivity and QoS support for a session is analyzed. In order to make realistic assumptions about the traffic distribution on the Internet, results from previous studies [53] were used, where it is shown that $25 \%, 40 \%, 26 \%$ and $9 \%$ of receivers are placed in a network at a distance of two, three, four and five networks from their source $(S 1)$, respectively. Four topologies were used (T1 to T4), where three receivers (R1 to R3) are connected to an access-agent-I (AA-I) and three receivers (R4 to R6) are linked to an access-agent-II $(A A-I I)$ as illustrated in Fig. 8. Both access-agents belong to the same network and the receivers are requesting the access to the session sequentially from R1 to R6. 


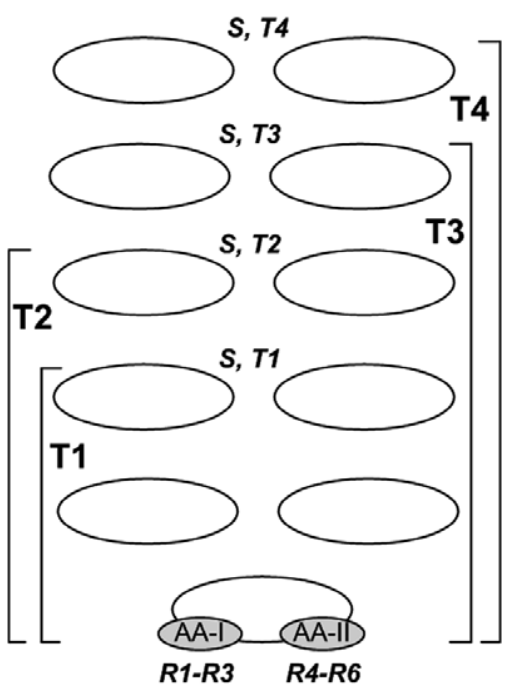

Fig. 8. Topologies used in the experiment.

Each experiment was repeated ten times and the distance between the receivers and the source was varied in terms of the number of networks (from 2 to 5). One SIPProxy was placed in the source's network and one in the receiver's access-network. First, SIP was used to allow the receivers to access the session (i.e., without MUSC) and, then, SIP was used together with MUSC following the MUSC proposal. Fig. 9 shows the average of the sum of the session setup times in each access-agent for the topologies T1, T2, T3 and T4.

The results revealed that the utilization of MUSC to allow the access of users to multi-user session reduces the time to establish the same session in $61 \%$ and $85 \%$ for receivers in $A A-I$ and $A A-I I$, respectively. These smaller setup times are possible because MUSC only exchanges end-to-end MUSC-P messages to setup the session for the first receiver in an access-agent, being all posterior requests processed locally in the access-agent. If the same flow of a session is already present in the ingress-agent of the access-network, which happens when receiver R4 requests the access to the session, the MUSC-P messages sent due to the request of $\mathrm{R} 4$ are transported only inside the access-network, and not end-to-end, as occurs with SIP to handle every request. The $95 \%$ confidence interval of the receiver's session setup time for each topology when SIP and MUSC approaches are used is summarized in Table 1.

Fig. 10 shows the results obtained to setup a session when there are four networks between the receivers and the source (with a $95 \%$ of confidence interval). The results show that in the worst case, the MUSC-P messages visit

Table 1

95\% Confidence interval of the session setup time for each topology

\begin{tabular}{lllll}
\hline Receiver & T1 $(\mathrm{ms})$ & $\mathrm{T} 2(\mathrm{~ms})$ & $\mathrm{T} 3(\mathrm{~ms})$ & $\mathrm{T} 4(\mathrm{~ms})$ \\
\hline R1-SIP & 0.89 & 0.97 & 0.90 & 1.11 \\
R1-MUSC & 0.84 & 1.02 & 0.77 & 0.87 \\
R2-SIP & 0.89 & 0.97 & 0.90 & 1.11 \\
R2-MUSC & 0.36 & 0.34 & 0.36 & 0.38 \\
R3-SIP & 0.89 & 0.97 & 0.90 & 1.11 \\
R3-MUSC & 0.36 & 0.34 & 0.36 & 0.38 \\
R4-SIP & 0.87 & 0.72 & 0.90 & 0.60 \\
R4-MUSC & 0.51 & 0.58 & 0.57 & 0.18 \\
R5-SIP & 0.87 & 0.72 & 0.90 & 0.60 \\
R5-MUSC & 0.33 & 0.38 & 0.19 & 0.29 \\
R6-SIP & 0.87 & 0.72 & 0.90 & 0.60 \\
R6-MUSC & 0.33 & 0.38 & 0.19 & 0.29 \\
\hline
\end{tabular}

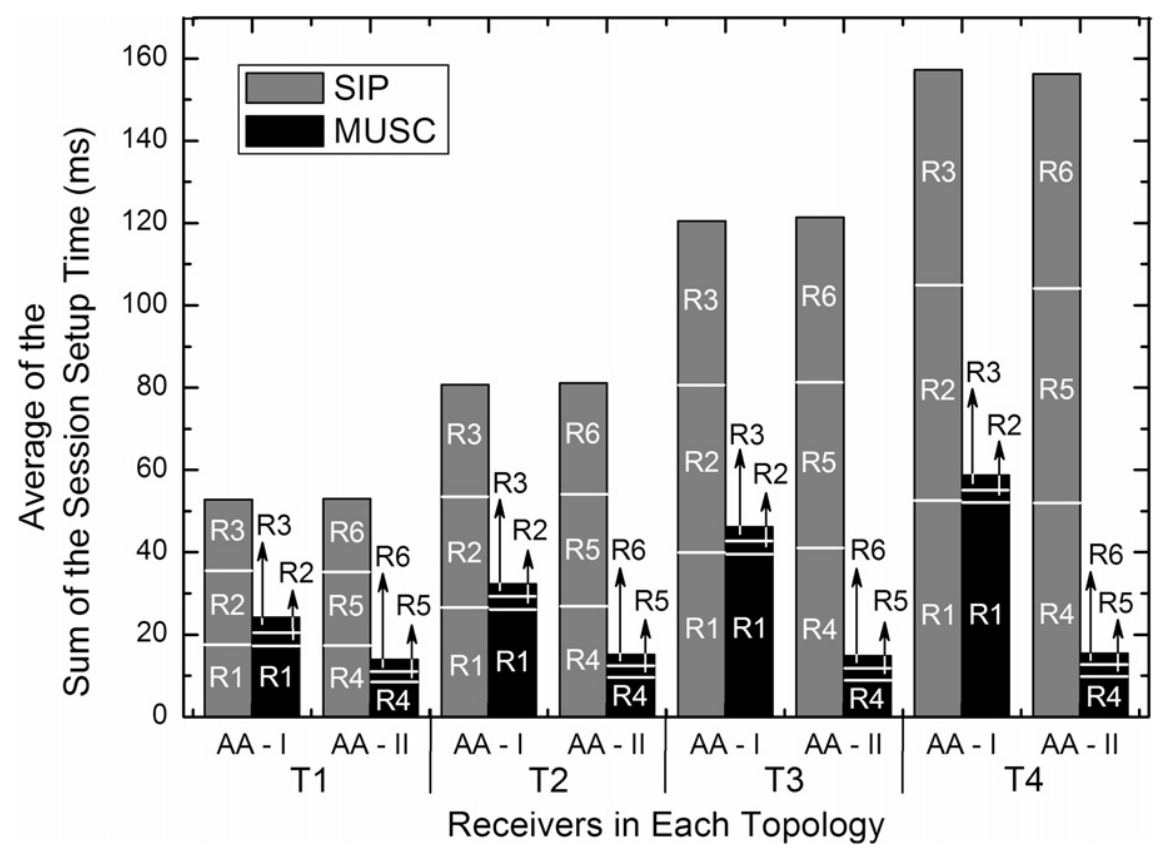

Fig. 9. Average of the sum of session setup times for SIP and MUSC. 


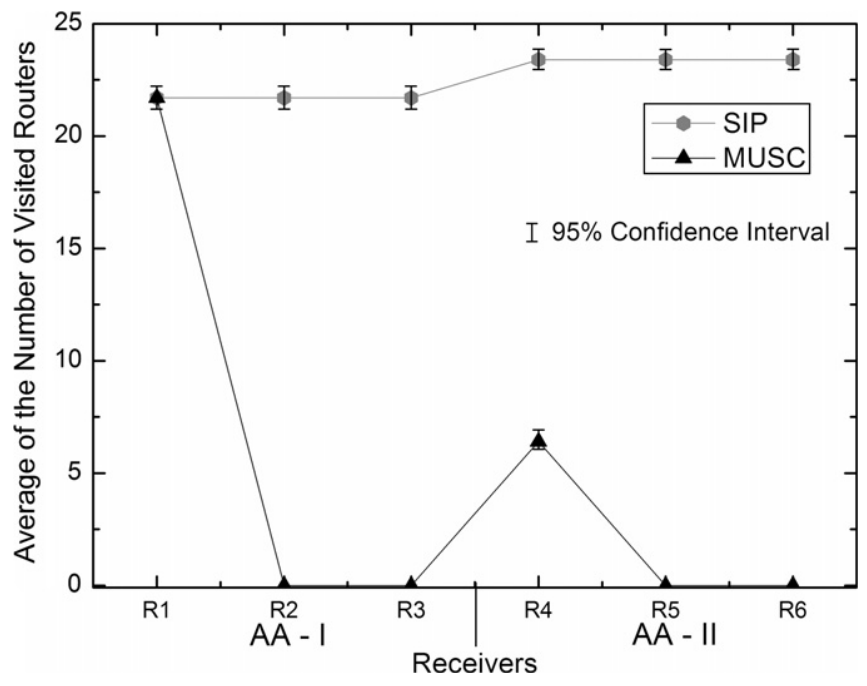

Fig. 10. Variation of the number of visited routers.

the same amount of routers than a SIP message. This situation occurs for the first receiver in an access-network. However, for the subsequent receivers in the same access-network, MUSC-P messages only travel locally inside the access-network, while SIP messages continue to have an end-to-end scope. This is illustrated for R4, where the MUSC-P messages visit $75 \%$ less routers than when only SIP is used. MUSC contributes to decrease the setup time as well as signaling and state overhead when setting-up the same session to several receivers in the same network. The difference between using MUSC or only SIP, in what concerns the number of visited routers, is even higher when subsequent receivers access a ses- sion in an access-agent in which another user of the same session is already attached. In this case there is no need to generate messages if MUSC is used, as shown by the examples of R2, R3, R5 and R6 in Fig. 10.

The next experiment addressed the evaluation of the session setup time when some QoS assurance is required for the sessions. The results are illustrated in Fig. 11 with a $95 \%$ confidence interval. The PIM-SSM is used to distribute packets in multicast-aware networks and DiffServ is implemented to provide packet differentiation on wired links, where the queues are served by the Weighted Fair Queuing (WFQ) discipline. In wireless links, MIRA is also configured to control network resources for IEEE 802.11e classes.

Fig. 11 illustrates that, on average, the total session setup time for receivers in $A A-I$ is higher than in $A A-I I$. This is caused by the interaction between MUSC and MIRA in all edge routers along the data path until the access-agent is reached, in order to setup the session for R1. However, when the session is ongoing in the accessnetwork and the first receiver of $A A-I I$ requests the access to the session, this interaction occurs only inside the accessnetwork. In addition, it shows that the inter-network operations have higher impact in the setup time than the overall intra-network ones. This is justified, because the MIRA inter-network resource reservation control does not require signaling exchanges. Moreover, inter-network links have higher propagation delay and MUSC needs time to configure the connectivity translators between the SSM channels used in different networks. Moreover, the setup time associated with MUSC includes the SIP delay between the endhost and the SIP server, and from the later to the accessagent.

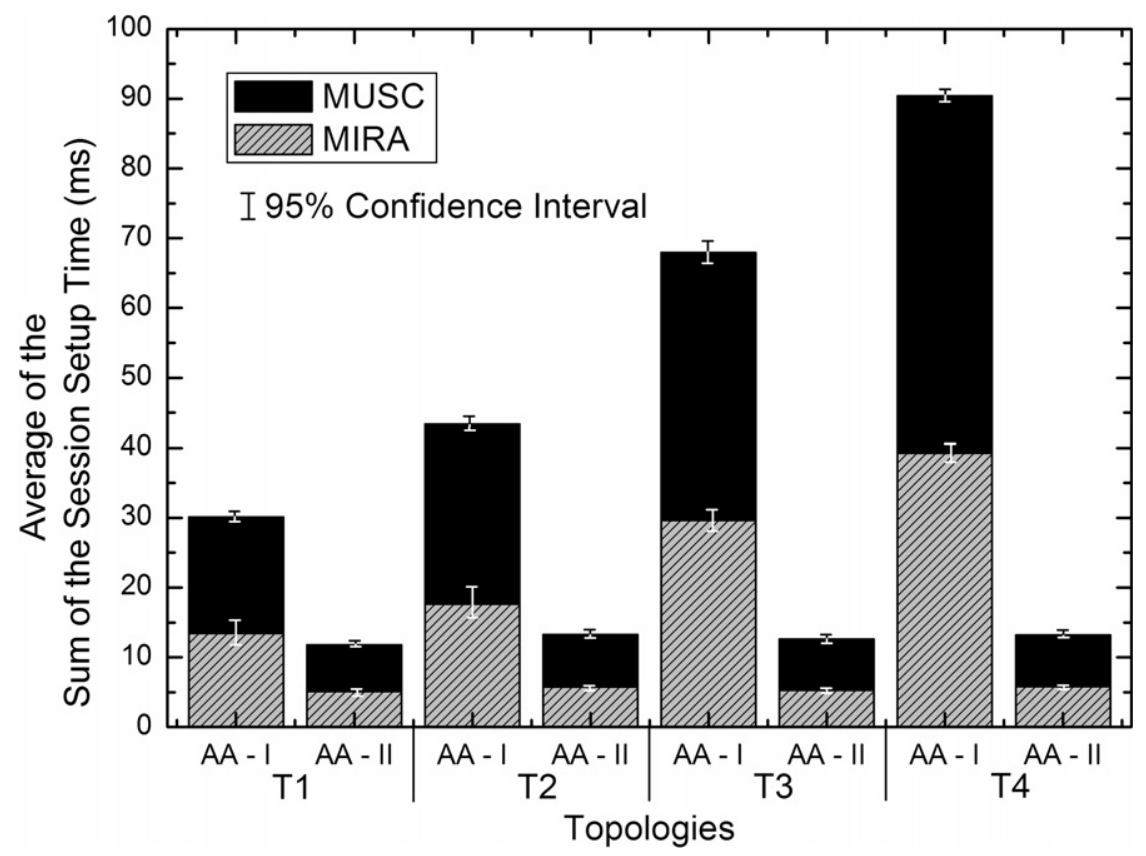

Fig. 11. Sum of session setup times for MUSC and MIRA. 
Additional simulation results concerning the efficiency of MUSC to setup ongoing sessions when SIP is used to control hard handovers are included in previous work [54]. The results show the impact in terms of packet delay and percentage of packet losses caused by the mobility of users inside and between networks.

\subsubsection{Seamless inter-network mobility management}

This simulation experiment analyzed the convergence time, signaling overhead and the impact on the percentage of packet lost due to an inter-network handover when MUSC is working together with SEMUD and MIRA. In this experiment, BRITE was used to generate a topology following the inter-network mobility scenario illustrated in Fig. 6. Two receivers in a multicast-aware access-network are connected to the same access-agent and get one Variable Bit Rate video with an average rate of $86 \mathrm{~KB} / \mathrm{s}$. PIM-SSM is used to distribute packets and DiffServ and IEEE 802.11e are configured to allow QoS assurance. The experiment assumed the existence of a mobility prediction scheme to trigger SEMUD to start the handover process in advance. After that, SEMUD transfers the session context and notifies MUSC in the predicted access-agent. This allows the pre-configuration of the session on a new path and the cache before its disconnection from the home network.

Table 2 presents the average convergence time of each component before and after the attachment of the moving receivers to the new access-agent. The convergence time

Table 2

Average session setup time (ms) used by SEMUD, MUSC and MIRA in an inter-network handover

\begin{tabular}{lllrlrl}
\hline $\begin{array}{l}\text { Mobile } \\
\text { receiver }\end{array}$ & \multicolumn{2}{l}{ Before handover } & & After & Total & $\begin{array}{l}95 \% \\
\text { Confidence } \\
\text { interval }\end{array}$ \\
\cline { 3 - 4 } & SEMUD & MUSC & MIRA & SEMUD & & \\
\hline R1 & 22.48 & 15.94 & 12.19 & 2.28 & 52.89 & 1.98 \\
$\mathrm{R} 2$ & 22.48 & - & 1.64 & 2.28 & 26.04 & 1.31 \\
\hline
\end{tabular}

before the attachment includes SEMUD, MUSC and MIRA signaling and their procedures to configure the cache, QoS mapping, connectivity, and network service, respectively, on the path towards the new access-agent. The convergence time after the attachment encompasses SEMUD operations to fetch the missing packets and to configure the multicast interface.

The results show that SEMUD requires more time to transfer the session context and to configure its mechanisms than MUSC or MIRA. The MUSC session setup time would be higher if the requested session for R1 would be activated in an agent near the source. The MIRA convergence time is small, because only intra-network operations are performed to reserve network resources in the selected class. Since the resources associated with the session requested by $\mathrm{R} 2$ are already configured on the path ending in the new access-agent, only MIRA QoS control operations are performed and the MUSC convergence time to allow the access of the receiver and to reply SEMUD is negligible. This functionality reduces in $60 \%$ the overall signaling overhead and in $49 \%$ the time required to setup the same session for a second receiver in the same access-agent as happens with R2. After handover, each receiver needs to wait $2.28 \mathrm{~ms}$ to receive packets associated with the ongoing session.

The use of SEMUD caching and buffering mechanisms supplies MUSC with seamless capability by reducing the impact of the mobility through the recovering of missing packets. Fig. 12 shows the benefits of the caching and buffering mechanisms in reducing the number of packet losses for a cache size of $100 \mathrm{~KB}$ and for a buffer size varying between 1 and $100 \mathrm{~KB}$. The depicted results were obtained for several values of the handover time when the mechanism was disabled and enabled. The handover time is the period between the disconnection from the old agent and the reception of the first packet in the foreign agent. For instance, the use of buffering and caching mechanisms allows the recovery of 21 packets when the handover time is $300 \mathrm{~ms}$ and the buffer size is $50 \mathrm{~KB}$. On the other hand,

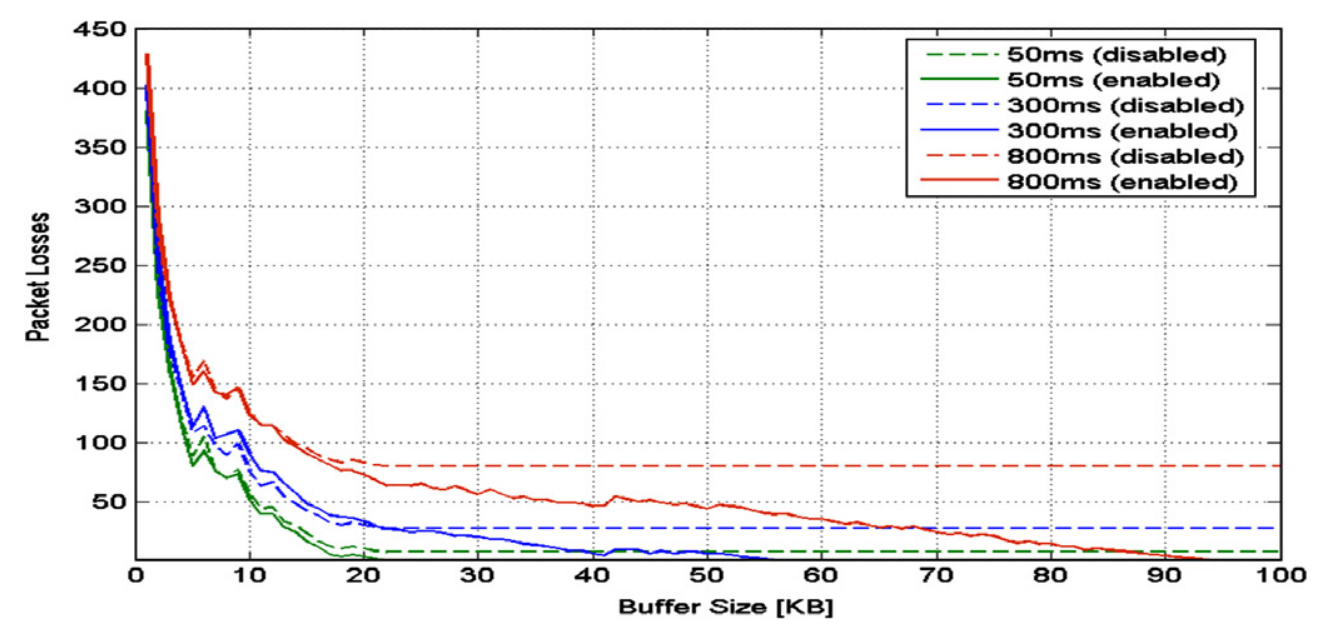

Fig. 12. Number of recovered packets for different cache sizes and handover times. 
for a handover duration of $800 \mathrm{~ms}, 80$ packets are lost in an experiment when the buffering and caching mechanisms are disabled, while nine packets are recovered when the buffer size is $85 \mathrm{~KB}$ (representing a reduction of $89 \%$ in the percentage of packet losses). In addition, to avoid packet losses during a handover of $300 \mathrm{~ms}$, the buffer size must be higher than $56 \mathrm{~KB}$. Hence, the benefits of the proposed buffering and caching mechanisms are evident.

\subsection{Prototype experiments}

In order to verify the MUSC QoS adaptation solution due to an inter-network hard handover in an experimental environment, a prototype test-bed was developed. The testbed is composed by three access networks, one source placed in one access network, one mobile receiver connected to another access network and five routers with MUSC and MIRA agents (three inter-network routers and two access-point routers following the scenario used in Fig. 7). All devices are Pentium IV $1.7 \mathrm{GHz}$ and use a Linux Fedora 2.6.16 kernel. The core routers are equipped with network interface cards at $10 \mathrm{Mb} / \mathrm{s}$, while the access routers support also a PCI wireless card IEEE $802.11 \mathrm{~b} / \mathrm{g}$ with an external antenna and a transmission rate of $11 \mathrm{Mb} / \mathrm{s}$. The source and the receiver are equipped with $11 \mathrm{Mb} / \mathrm{s}$ IEEE 802.11b/g network card. The Distributed Internet Traffic Generator (D-ITG) is responsible to send and to receive flows of the session and the mobility is controlled by MIPv4 bidirectional tunneling.

Each session is sent from one source and is composed of three flows with the same QoS requirements. Although MUSC can handle any number of flows, three flows allow a good trade-off between quality and bandwidth, and additional flows only provide marginal improvements [55]. Additionally, each flow has different priorities and exponential rates, which are common in scalable CODECS [55]. Each one of the three flows has a Constant Bit Rate (CBR) of 32, 64 and $128 \mathrm{~Kb} / \mathrm{s}$, starting from the most important to the less important one. It is assumed that intolerance to loss is the major requirement and that a loss limit of $2.5 \%$ is the maximum degradation allowed in the QSPEC. This limit is based on previous studies [56], where it is presented that in MPEG-2 with Signal-to-Noise Ratio scalability, $5 \%$ of losses in the most important flow introduces $100 \%$ of losses in all other flows. The QSPEC object of each flow of a session is generated following a Poisson distribution.

Since inter-network adaptation is the focus of this evaluation, the bandwidth required for the session exceeds the amount of resources assigned to the preferred class in the inter-network link from the access network hosting the source and the receiver's foreign network. Consequently, MUSC is notified by MIRA to adapt the session to the current network conditions, while avoiding the session blocking and keeping ongoing session with an acceptable quality level. The following QoS adaptation profiles are used to evaluate the MUSC QoS control proposal: (i) The $A D P \_$Drop profile, in which session quality is controlled by dropping and adding flows. (ii) The $A D P \_H y b$ profile maps flows with high priority into the preferred service class and flows with lower priority are re-allocated to a less important class. (iii) The $A D P \_$Sub profile maps all flows of a session to a less important class, avoiding the packet-reordering and assuring the session full rate.

In each experiment, the receiver moves to the foreign network $45 \mathrm{~s}$ after its subscription and returns to the home network $65 \mathrm{~s}$ latter. Fig. 13(a) shows the average throughput measured in the receiver when the ADP_Drop, $A D P \_H y b$ and $A D P \_S u b$ profiles are used. Furthermore, Fig. 13(b) shows in detail the average throughput measured by the receiver when $A D P \_S u b$ profile is being used.

The proposed QoS control scheme introduces an average latency of $1 \%$ to reserve resources and to configure mapping and adaptation mechanisms during the mobility process, which can be considered negligible. In addition, the results reveal that MUSC assures the session full rate

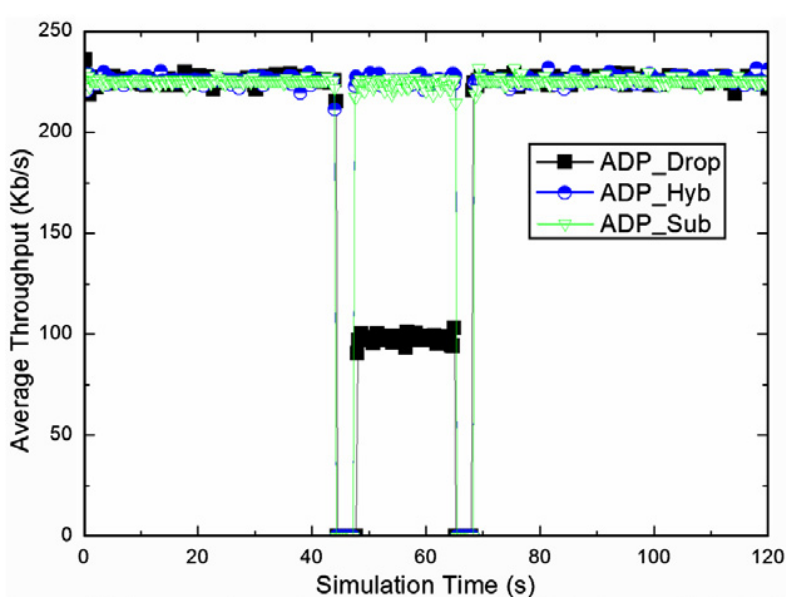

(a) Average throughput and latency for ADP_Drop, ADP_Hyb and ADP_Sub profiles

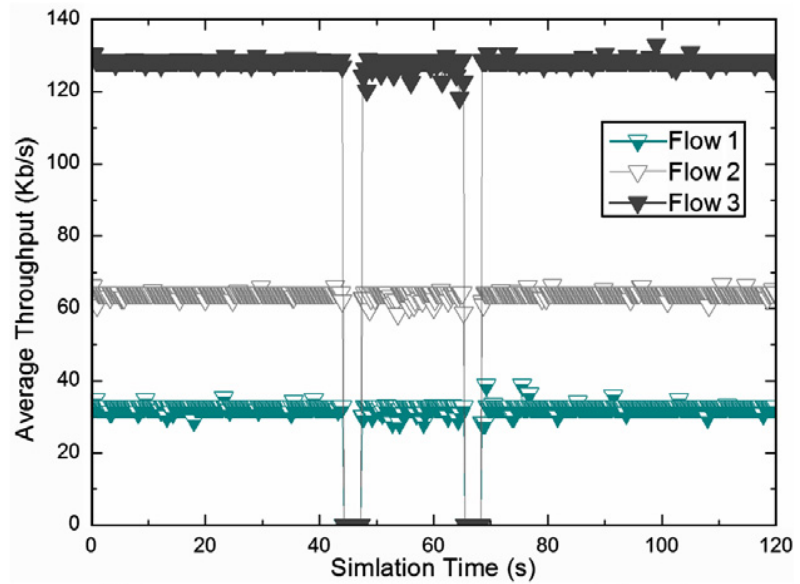

(b) Average throughput and latency for each flow of the session when ADP Sub profile is used

Fig. 13. Average throughput observed by the receiver. 
Table 3

Throughput in home and foreign network for all profiles

\begin{tabular}{llll}
\hline Throughput & $\begin{array}{l}\text { ADP_Drop } \\
(\mathrm{Kb} / \mathrm{s})\end{array}$ & $\begin{array}{l}\text { ADP_Hyb } \\
(\mathrm{Kb} / \mathrm{s})\end{array}$ & $\begin{array}{l}\text { ADP_Sub } \\
(\mathrm{Kb} / \mathrm{s})\end{array}$ \\
\hline Home - Max & 229.46 & 229.40 & 229.98 \\
Home - Min & 218.99 & 219.42 & 219.06 \\
Home - Med & 224.51 & 224.98 & 224.49 \\
Foreign - Max & 103.88 & 228.08 & 226.84 \\
Foreign - Min & 89.50 & 216.78 & 217.02 \\
Foreign- Med & 96.17 & 223.79 & 222.48 \\
\hline
\end{tabular}

(flows $F 1, F 2$ and $F 3$ with approximately 32, 64 and $128 \mathrm{~Kb} / \mathrm{s}$, respectively, during the whole experiment) when $A D P \_H y b$ and $A D P \_S u b$ are configured, by using resources available in other service classes. The profile $A D P$ _Drop controls the session quality level and keeps it with an acceptable level, because only the less important flow of the session is affected in the foreign network. With any of the profiles, the session full rate is guaranteed in the home network, since there are resources to accommodate the flows in the preferred class. The throughput is zero in the time intervals $[\sim 44 \mathrm{~s}, 47.35 \mathrm{~s}]$ and $[\sim 65 \mathrm{~s}, 68.25 \mathrm{~s}]$ since the receiver is moving to the foreign network and returning to its home network, respectively. The standard deviation of the handover latency is $\sim 0.8 \mathrm{~s}$ and the $95 \%$ confidence interval is $\sim 0,51 \mathrm{~s}$. Table 3 summarizes the results obtained with each used profile, by measuring the throughput when the receiver is in the home and foreign networks.

As explained before, the full rate of ongoing sessions is assured by the $A D P \_H y b$ and $A D P \_S u b$ profiles, while the RTT is degraded. On average, the RTT in the home network, where the session is mapped into the preferred class, is of $1.98 \mathrm{~ms}$. In the foreign network, the average RTT is increased, due to the encapsulation/decapsulation of packets inherent to the MIP bidirectional tunneling approach. In addition to the time consumed by the QoS-aware tunnels, the session adaptation to a service class that offers different delay tolerance also influences the RTT. Thus, the receiver needs to wait, on average, $3.45,3.67$ and $3.81 \mathrm{~ms}$ when the $A D P \_$Drop, $A D P \_H y b$ and $A D P \_S u b$ profiles are used, respectively. This means that in the foreign network, the RTT delay is increased in 174\% when ADP_Drop is configured. However, the delay is increased in $185 \%$ for $A D P \_H y b$ and $192 \%$ for ADP_Sub profiles.

\section{Conclusion and future work}

This article presents the MUSC mechanism to control the access and mobility of multi-user sessions across heterogeneous networks. The interaction with SEMUD enhances MUSC with seamless mobility capability and the communication with MIRA allows the construction of QoS-aware distribution trees for fixed and mobile users independently of the QoS model, service classes and current network conditions on the path from the sender to the receivers. An interface with MIP allows MUSC to control the quality level of ongoing sessions with QoS support.
MUSC has open interfaces, allowing operators to use resource allocation and mobility controllers of their choice.

Simulation experiments showed the session setup time reductions brought by MUSC, when compared to a solution using only SIP. MUSC only needs local operations to allow a second receiver to access an already activated session in the same access-network, which minimizes the convergence time and signaling overhead. Moreover, the impact caused when network resource reservations and seamless mobility operations are performed is also introduced. An example of the efficiency of the MUSC scheme supplied with seamless mobility is a reduction of $75 \%$ of packet losses for a cache and buffer sizes of 50 and $55 \mathrm{~KB}$, respectively.

The prototype experiments showed the operation of MUSC to control the quality level of an ongoing session due to an inter-network handover of its receiver. The variation of the average throughput measured in the receiver, when it handovers from its home to the foreign network depends upon the adaptation profile used: it was kept in its full rate by the $A D P_{-} H_{y} b$ and $A D P \_S u b$ (around $223 \mathrm{~Kb} / \mathrm{s}$ ) and it decreased in 57\% when $A D P \_$Drop was used. Nevertheless, the lower throughput imposed by the $A D P$ Drop scheme still provides an acceptable quality level to the session, since less significant flows are the first to be dropped. The session RTT is increased in the foreign network due to the creation of MIP tunnels and it is also influenced by the QoS profile used in the system, where the $A D P \_S u b$ is expected to introduce the highest RTT since the class selected by MUSC was more tolerance to delay. The measurements made in the prototype provide hints about the most efficient adaptation schemes. The measured combined performance of ADP_Drop, $A D P \_H y b$ and $A D P \_S u b$ methods was of $[57 \%, 174 \%],[99.5 \%, 185 \%]$ and $[99.2 \%, 192 \%]$, respectively, being the parameters related to the average throughput (first) and RTT (second) variations.

A heuristic approach for the combination of all adaptation profiles according to historical data and traffic patterns will be investigated. MUSC will also be analyzed together with different mobility prediction techniques to verify the impact of MUSC operation in the handover times.

\section{Acknowledgements}

This work was done at the Laboratory of Communications and Telematics of the Faculty of Science and Technology of the University of Coimbra. It is supported by DoCoMo Euro-labs, by the Portuguese Ministry of Science, Technology and High Education, and by European Union FEDER - POSC (projects Q3M and SAPRA).

\section{References}

[1] B. Quinn, K. Almeroth, IP Multicast Applications: Challenges and Solutions, IETF RFC 3170, September 2001. 
[2] T. Shanableh, M. Ghanabari, Multilayer transcoding with format portability for multicasting of single-layered video, IEEE Transaction on Multimedia 7 (1) (2005).

[3] M. Carugi, B. Hirschman, A. Narita, Introduction to the ITU-T NGN focus group release 1: target environments, services and capabilities, IEEE Communication Magazine 43 (10) (2005).

[4] V. Pereira, P. Mendes, E. Monteiro, Evaluation of an overlay for source-specific multicast in asymmetric routing environments, in: Proceedings of IEEE Global Telecommunications, Washington, USA, November 2007.

[5] J. Babiarz, K. Chan, F. Baker, Configuration Guidelines for DiffServ Service Classes, IETF RFC 4594, August 2006.

[6] C. Perkins, J. Arkko, IP Mobility Support for IPv4, IETF RFC 3220, January 2002.

[7] J. Rosenberget et al., SIP: Session Initiation Protocol, IETF RFC 3261, June 2002.

[8] L. Zhou, Y. Sun, An analysis of multicast support for mobile hosts using mobile IPv6, in: Proceedings of IEEE International Conference on Wireless Communications, Networking and Mobile Computing, Wuhan, China, September 2006.

[9] E. Cerqueira et al., Multi-user session control in the next generation wireless system, in: Proceedings of ACM International Workshop on Mobility Management and Wireless Access, Malaga, Spain, October 2006.

[10] L. Veloso et al., Seamless mobility of users for media distribution services, in: Proceedings of IEEE International Performance Computing and Communications Conference, New Orleans, USA, April 2007

[11] A. Neto et al., Resource reservation protocol supporting QoS-aware multicast trees for next generation networks, in: Proceedings of IEEE Symposium on Computers and Communications, Aveiro, Portugal, July 2007.

[12] H. Schulzrinne et al., Real Time Streaming Protocol 2.0 (RTSP), IETF RFC 2326, April 1998.

[13] D. Johnson, C. Perkins, J. Arkko, Mobility Support in IPv6, IETF RFC 3775, June 2004.

[14] H. Soliman et al., Hierarchical Mobile IPv6 Mobility Management (HMIPv6), IETF RFC 2140, August 2005.

[15] R. Koodli, Fast Handovers for Mobile IPv6, IETF RFC 4068, July 2005.

[16] C. Lin, K.-M. Wang, Mobile multicast support in IP networks, in: Proceedings of IEEE Annual Joint Conference of the Computer and Communications Societies, Tel-Aviv, Israel, March 2000.

[17] T. Schmidt, M. Waehlisch, Multicast Mobility in MIPv6: Problem Statement and Brief Survey, IETF Internet Draft, November 2007.

[18] J. Hillebrand et al., Quality-of-Service Management for IP-Based Mobile Networks, in: Proceedings of IEEE Wireless Communications and Networking Conference, New Orleans, USA, March 2005.

[19] I. Miloucheva, Context management for efficient mobile multicast services, in: Proceedings of International Workshop on Context in Mobile Human Computer Interfaces, Las Vegas, USA, September 2005.

[20] B. Zhang et al., Host multicast: a framework for delivering multicast to end users, in: Proceedings of Annual Joint Conference of the IEEE Computer and Communications Societies, New York, USA, June 2002.

[21] E. Pearce et al., System and Method for Enabling Multicast telecommunications, Patent, US7079495 B1, July 2006.

[22] Z. Jumbiao, Multicast over Unicast in a Network, Patent, MX2006PA03857, July 2006.

[23] R. Schantz et al., Controlling quality-of-service in distributed realtime and embedded systems via adaptive middleware: experiences with auto-adaptive and reconfigurable systems, in: IEEE Transaction on Software Practice \& Experience, vol. 36, New York, USA, September 2006.

[24] M. Ruy et al., QoS class mapping over heterogeneous networks using Application Service Map, in: Proceedings of IEEE International Conference on Networking, International Conference on Systems and
International Conference on Mobile Communications and Learning Technologies, Washington, USA, April 2006.

[25] Z. Mammeri, Approach for end-to-end QoS mapping and handling, in: Proceedings of IEEE/IFIP International Conference on Wireless and Optical Communications Networks, Dubai, United Arab Emirates, March 2005.

[26] S. Johansen, A. Kim, A. Perkis, Quality incentive assisted congestion control for receiver-driven multicast, in: Proceedings of the IEEE International Conference on Communications, Glasgow, UK, June 2007.

[27] J. Kim et al., Efficient video transcoding technique for QoS-based home gateway service, IEEE Transactions on Consumer Electronics 52 (1) (2006).

[28] H. Schulzrinne, R. Hancock, GIST: General Internet Signalling Transport, IETF Internet Draft, July 2007.

[29] J. Ash et al., QoS NSLP QSPEC Template, IETF Internet Draft, (in press).

[30] H. Chaskar, Requirements of a Quality of Service (QoS) Solution for Mobile IP, IETF RFC 3583, September 2003.

[31] T. Sanda et al., Applicability Statement of NSIS Protocols in Mobile Environments, IETF Internet Draft, November 2007.

[32] H. Jang et al., Mobile IPv6 Fast Handovers over IEEE 802.16e Networks, IETF Internet Draft, November 2007.

[33] E. Duros et al., A Link Layer Tunnelling Mechanism for Unidirectional Links, IETF RFC 3077, March 2001.

[34] I. Miloucheva, J. Moedeker, D. Hetzer, Handover and resource management of mobile node with unidirectional links, in: Proceedings of IEEE International Conference on Wireless and Mobile Communications, Guadeloupe, French Caribbean, March 2007.

[35] M. Liebsch et al., Candidate Access Router Discovery (CARD), IETF RFC 4066, July 2005.

[36] A. Roy, S.K. Das, A. Misra, Exploiting information theory for adaptive mobility and resource management in future cellular networks, IEEE Wireless Communications 11 (4) (2004).

[37] T. Poon, E. Chan, Traffic management in wireless ATM network using a hierarchical neural-network based prediction algorithm, in: Proceedings of International Conference on Computers and their Applications, New Orleans, USA, March 2000.

[38] M. Sricharan et al, An activity based mobility prediction strategy for next generation wireless networks, in: Proceedings of IFIP Conference on Wireless and Optical Communications Networks, Bangalore, India, April 2006.

[39] S. Michaelis, C. Wietfeld, Comparison of user mobility pattern predication algorithms to increase handover trigger accuracy, in: Proceedings of IEEE Vehicular Technology Conference, Montreal, Canada, September 2006.

[40] IEEE 802.16-2004 Specification, 802.16-2004 IEEE Standard for Local and Metropolitan Area Networks, Part 16: Air Interface for Fixed Broadband Wireless Access Systems, October 2004.

[41] IEEE 802.16e-2005 Specification, 802.16e-2005 IEEE Standard for Local and Metropolitan Area Networks, Part 16: Air Interface for Fixed Broadband Wireless Access Systems-Amendment 2: Physical and Medium Access Control Layers for Combined Fixed and Mobile Operation in Licensed Bands, February 2006.

[42] X. Chen, et al., Supporting QoS in IEEE 802.11e Wireless LANs, IEEE Transaction on Wireless Communications, vol. 5, No. 8, August 2006.

[43] J. Moy, OSPF Version 2, IETF RFC 2328, April 1998.

[44] Y. Rekhter, T. Li, A Border Gateway Protocol 4 (BGP-4), IETF RFC 1771, March 1995.

[45] H. Agrawal, M. Grah, M. Gregory, Optimization of QoS routing, in: Proceedings of IEEE International Conference on Computer and Information Science, Melbourne, Australia, July 2007.

[46] P. Mendes, J. Andres-Colas, C. Pinho, Information model for the specification of QoS agreements among ambient networks, in: Proceedings of IEEE Symposium on Personal Indoor and Mobile Radio Communications, Berlin, Germany, September 2005. 
[47] M. Handley, V. Jacobson, C. Perkins, SDP: Session Description Protocol, IETF RFC 4566, July 2006.

[48] B. Cain et al., Internet Group Management Protocol, Version 3, IETF RFC 3376, October 2006.

[49] R. Vida, L. Costa, Multicast Listener Discovery Version 2 (MLDv2), IETF RFC 3810, June 2004.

[50] V. Fajardo, J. Arkko, J. Loughney, Diameter Based Protocol, IETF RFC 3588, September 2003.

[51] M. Garcia-Martin et al., Diameter Session Initiation Protocol (SIP) Application, IETF RFC 4740, November 2006.

[52] J. Korhonen et al., Diameter Mobile IPv6: Support for Home Agent to Diameter Server Interaction, IETF Internet Draft, November 2007.
[53] U. Steve et al., Implications of interdomain traffic characteristics on the traffic engineering, in: Proceedings of ACM Symposium on Applied Computing, Nicosia, Cyprus, March 2004.

[54] E. Cerqueira et al., Mobility support for multi-user sessions over heterogeneous networks, in: Proceedings of IEEE Symposium on Computers and Communications, Aveiro, Portugal, July 2007.

[55] K. Rose, S. Regunathan, Toward optimality in scalable predictive coding, IEEE Transaction on Image Processing 7 (2001).

[56] J. Kimura et al., Perceived quality and bandwidth characterization of layered MPEG-2 video coding, in: Proceedings of SPIE International Symposium, USA, September 1999. 